\title{
Upscaling in Vertically Fractured Oil Reservoirs Using Homogenization
}

\author{
Hamidreza Salimi • Hans Bruining
}

Received: 21 May 2009 / Accepted: 29 September 2009 / Published online: 22 October 2009

(C) The Author(s) 2009. This article is published with open access at Springerlink.com

\begin{abstract}
Flow modeling in fractured reservoirs is largely confined to the so-called sugar cube model. Here, however, we consider vertically fractured reservoirs, i.e., the situation that the reservoir geometry can be approximated by fractures enclosed columns running from the base rock to the cap rock (aggregated columns). This article deals with the application of the homogenization method to derive an upscaled equation for fractured reservoirs with aggregated columns. It turns out that vertical flow in the columns plays an important role, whereas it can be usually disregarded in the sugar cube model. The vertical flow is caused by coupling of the matrix and fracture pressure along the vertical faces of the columns. We formulate a fully implicit three-dimensional upscaled numerical model. Furthermore, we develop a computationally efficient numerical approach. As found previously for the sugar cube model, the Peclet number, i.e., the ratio between the capillary diffusion time in the matrix and the residence time of the fluids in the fracture, plays an important role. The gravity number plays a secondary role. For low Peclet numbers, the results are sensitive to gravity, but relatively insensitive to the water injection rate, lateral matrix column size, and reservoir geometry, i.e., sugar cube versus aggregated column. At a low Peclet number and sufficiently low gravity number, the effective permeability model gives good results, which agree with the solution of the aggregated column model. However, ECLIPSE simulations (Barenblatt or Warren and Root (BWR) approach) show deviations at low Peclet numbers, but show good agreement at intermediate Peclet numbers. At high Peclet numbers, the results are relatively insensitive to gravity, but sensitive to the other conditions mentioned above. The ECLIPSE simulations and the effective permeability model show large deviations from the aggregated column model at high Peclet numbers. We conclude that at low Peclet numbers, it is advantageous to increase the water injection rate to improve the net present value. However, at high Peclet numbers, increasing the flow rate may lead to uneconomical water cuts.
\end{abstract}

H. Salimi $(\varangle) \cdot$ H. Bruining

Department of Geotechnology, Delft University of Technology, Stevinweg 1, 2628 CN Delft,

The Netherlands

e-mail: h.salimi@tudelft.nl 
Keywords Upscaling · Fractured reservoirs · Homogenization · Waterflooding ·

Oil recovery

\section{List of Symbols}

A Horizontal cross-section

$B \quad$ 3D domain

c Vector of the matrix-cell center

d Size of a grid cell

$D_{\text {cap }}$ Capillary-Diffusion coefficient

e Unit normal vector

$F \quad$ Nonlinear fracture function

$F_{1,2} \quad$ Fracture set

$g \quad$ Gravity acceleration

$H \quad$ Height of the reservoir

I Unit tensor

$k \quad$ Permeability

$k_{\mathrm{f}} \quad$ Effective fracture permeability

$k_{\mathrm{r}} \quad$ Relative permeability

$l \quad$ Local scale (lateral matrix column size)

$L \quad$ Global scale/length of the reservoir

$M \quad$ Nonlinear matrix function

n Unit normal vector

$N_{\mathrm{f}} \quad$ Number of fracture grid cells

$N_{\mathrm{G}} \quad$ Gravity number

$N_{\mathrm{m}} \quad$ Number of matrix grid cells

$N_{\text {zf }} \quad$ Number of fracture grid cells in the vertical direction

p Vector of the fracture-cell center

$P \quad$ Pressure

$p_{\mathrm{c}} \quad$ Capillary pressure

$\mathbf{p}^{\prime} \quad$ Vector of the fracture-cell center on a horizontal cross-section

$\mathrm{Pe} \quad$ Peclet number

PV Pore volume

q Any parameter

$Q \quad 3$ D domain

$q_{\text {ext }}$ External (injection/production) rates

$q_{\mathrm{w}} \quad$ Water injection rate

r Coordinate vector

$R \quad$ Real

$S \quad$ Saturation

$S_{\text {or }} \quad$ Residual oil saturation

$S_{\mathrm{wc}} \quad$ Connate water saturation

SU Small unit

$t$ Time

$u \quad$ Velocity

u Velocity vector

$W \quad$ Width of the reservoir

$x \quad x$-coordinate 
$\mathbf{x}_{\mathrm{b}}$ Global coordinate

$\mathbf{x}_{\mathrm{p}}$ Center of a grid cell

$\mathbf{x}_{\mathrm{s}}$ Local coordinate

$x^{\prime} \quad$ Horizontal cross-section position

$y \quad y$-coordinate

$z \quad$ Vertical upward direction

Z 1D domain

\section{Greek}

$\alpha$ Phase (oil/water)

$\varepsilon \quad$ Scaling ratio

$\lambda$ Mobility

$\mu$ Viscosity

$\xi$ Potential/saturation indicator

$\rho$ Density

$\sigma$ Coordinates of the boundary

$\varphi$ Porosity

$\Phi$ Potential

$\Omega$ Horizontal cross-section domain

$\omega$ Auxiliary function

\section{Math Signs and Operator}

<〉 Average sign over volume

II Absolute value of volume

$\approx$ Almost equal to

$\otimes \quad$ Dyadic product

$\sqrt{ }$ Square root

$\int$ Integral

$\rightarrow$ Vector sign

d Differential

$\partial_{\pi} \quad$ Partial differential with respect to $\pi$

$\partial \quad$ Boundary of

$\nabla \quad$ Del (gradient operator)

$\Delta \quad$ Delta (difference operator)

$\nabla \quad$ Divergence operator

\section{Subscripts}

b Global (big) index

D Dimensionless

f Fracture

m Matrix

o Oil phase

$r$ Relative

$\mathrm{R}$ Reference

s Local (small) index

w Water phase 
$z \quad z$-coordinate (vertical direction)

$\alpha \quad$ Oil/water index

$\zeta$ Fracture/matrix index

\section{Superscripts}
* Local fracture index
(0) Zeroth-order index
(1) First-order index
(2) Second-order index

\section{Introduction}

Fractured hydrocarbon reservoirs provide over $20 \%$ of the world oil reserves and production (Firoozabadi 2000). A naturally fractured reservoir (NFR) is a reservoir that contains fractures that result from natural, as opposed to man-made, stress differences that existed in the rock at the time it fractured. These natural fractures can have a positive, neutral, or negative effect on fluid flow (Nelson 1985; Aguilera 1998). Virtually, all reservoirs contain at least some natural fractures (Aguilera 1998). However, if the effect of these fractures on fluid flow is negligible (neutral), the reservoir can, from a reservoir-engineering point of view, be treated as a single-porosity conventional reservoir (Chen 2007). We only consider reservoirs where fluid flow occurs predominantly in a fracture network and do not consider the case that fractures act as a barrier for fluid flow.

There are many mechanisms that cause fracturing of reservoirs, which are listed in Nelson (1985). Natural fractures are classified into diagenetic, tectonic, and regional. Diagenetic fractures are caused by physical and or chemical changes in the rock and can have any arbitrary orientation. Tectonic fractures are created due to a local tectonic event. Because of dominance of the vertical stress, fractures will develop perpendicular to the bedding plane. Finally, regional fractures are always perpendicular to major bedding surfaces and develop over large areas of the earth's crust with relatively little change in orientation. From the topological point of view, we can distinguish reservoirs that are built from matrix blocks that are bounded by fracture planes in all directions (sugar cube) or are bounded only by more or less vertical fracture planes (aggregated columns). Matrix blocks are pressed against each other, and consequently contact regions are usually crushed and impermeable. The top and bottom of these columns are bounded by the impermeable cap and base rock.

Depending on the mechanism, the fractured reservoirs can be classified into three different groups (Aguilera 1998; Firoozabadi 2000). For group A, the bulk of the hydrocarbon resides in the matrix, and the fracture pore volume (PV) is small in comparison to the matrix PV. NFR's of group B have about equal storage capacity in matrix and fractures, and in NFR's of group $\mathrm{C}$, the storage capacity is entirely in the fractures. In this work, we obtain upscaled equations for vertically fractured reservoirs of group A.

Fractured reservoirs simulations completely differ from conventional reservoirs simulations. The key issue for simulating flow in fractured reservoirs is how to handle the fracture-matrix interaction under different conditions (Wu et al. 2004). This is because the fracture-matrix interaction leads to a delayed response that distinguishes the flow through fractured reservoirs from the flow through heterogeneous single-porosity reservoirs. Consequently, there are fundamental differences between recovery performance of fractured and unfractured reservoirs. Capillary forces, leading to counter-current or co-current imbibition 
(Pooladi-Darvish and Firoozabadi 2000), are the main drivers for recovery from the matrix blocks (Firoozabadi 2000). However, gravity forces cannot be completely disregarded.

As said above, many geological situations lead to some type of fractured reservoirs. The modeling of flow in both the random and correlated fracture network has attracted considerable attention in the hydrology community. Margolin et al. (1998) examined the interplay and relative importance of the fracture structure and fracture aperture variation. Berkowitz et al. (2001) examined a set of analytical solutions based on the continuous time random walk approach to analyze breakthrough data for tracer test in fractures and heterogeneous porous media. Park et al. (2001) assessed the importance of fracture intersection mixing rules on simulated solute migration patterns in random fracture network. Moreover, De Dreuzy et al. $(2002,2004)$ described the influence of the fracture length and fracturecenter correlation pattern on the equivalent permeability of the random fracture network. However, the current literature in the petroleum community dealing with flow in fractured reservoirs is largely confined to topological equivalents of the sugar cube model, i.e., the matrix blocks are surrounded by fractures from all sides (Barenblatt et al. 1960; Warren and Root 1963; Kazemi et al. 1969; Sonier et al. 1988; Kazemi et al. 1992; Al-Huthali and DattaGupta 2004; Di Donato and Blunt 2004; AI-Harbi et al. 2005; Sarma and Aziz 2006). All these paper use the semi-empirical transfer function approach of the BWR method. However, the aggregated column model is topologically different because it is not connected to the fracture network through the top and bottom of the matrix column. To our knowledge, the current reservoir simulators have no option to deal with this situation and this is the innovative aspect of this contribution. Indeed, the physics of the aggregated column model is completely different from the physics of the sugar cube model. The main reason is that gravity plays a more important role, meaning that gravity and capillary phenomena interact in the matrix columns.

We assert that the upscaling problem for aggregated columns can be tackled using homogenization. It turned out that homogenization first developed by Tartar (1980) and summarized by Hornung (1997) was suitable for upscaling fractured reservoirs and applied to fractured media by Arbogast et al. (1990), Arbogast (1993a, b), and Douglas et al. (1991). Hoteit and Firoozabadi (2006) developed discontinuous Galerkin and mixed finite-element methods to solve the ensuing model equations. Homogenization is a powerful upscaling technique, which has been successfully applied to a variety of problems of interest, such as reactive contaminant transport (Lewandowska et al. 2005; Van Duijn et al. 2008) and two-phase flow in layered (Van Duijn et al. 2002) and fractured media (Douglas et al. 1993; Chen 2007). It has several advantages over other upscaling techniques, such as representative elementary volume (REV) averaging; it does not use intuitive closure equations and it explicitly shows the dependence of the upscaled equations on the characteristic dimensionless numbers of interest. However, homogenization uses a number of assumptions for the physics of flow in porous media. All these assumptions need to be validated from the physical point of view. Therefore, we also give a full overview of simplifications and assumptions needed for the aggregated columns to justify the upscaling procedure. In this case, only upscaling in a plane perpendicular to the columns is required.

We use the computed results from the upscaled equations to investigate the effect of water injection rate, lateral matrix column size, and gravity on the cumulative oil production. Moreover, we compare the upscaled results for the aggregated columns with results obtained for sugar cube models described in a previous paper (Salimi and Bruining 2009), for various Peclet numbers (capillary diffusion time in matrix columns/residence time in fractures) and gravity numbers. Furthermore, we compare with results from the effective permeability model, which in principle is a conventional single-porosity model. In addition, we also investigate whether the aggregated column model can be solved with the current state of the art 
simulation programs, e.g., ECLIPSE simulations with a shape factor for which the vertical height is equal to the layer height.

The article is organized as follows. Section 2 describes the physical model and the geometrical configuration. Section 3 explains the upscaling technique (homogenization). In Sect. 4, we define characteristic dimensionless numbers. In Sect. 5, we derive the fully implicit numerical model. The results in Sect. 6 also include comparisons with the sugar cube model, the effective permeability model, and ECLIPSE. Finally, we summarize our conclusions.

\section{Physical Model}

We consider a vertically fractured network (Fig. 1) in the domain $Q=A_{\mathrm{Q}} \times Z$, where $A_{\mathrm{Q}}=\{x, y \in \mathrm{R} \mid 0 \leq x \leq L, 0 \leq y \leq W\}$ and $\mathrm{Z}=\{z \in \mathrm{R} \mid 0 \leq z \leq H\}$, where $\mathrm{R}$ represents the set of real numbers. We use $L, W$, and $H$ to denote the length, width, and height of the fractured reservoir, respectively. There are two mutually perpendicular vertical fracture sets $F_{1}, F_{2}$, which surround the matrix column $B_{\mathrm{m}}=\Omega_{\mathrm{m}} \times Z$, where $\Omega_{\mathrm{m}}$ (see Fig. 1c) denotes the domain of a horizontal cross-section of a matrix column. Therefore, matrix columns are connected from the top to the bottom of the reservoir domain, and there is no horizontal fracture connection between matrix columns. Thus, the matrix columns can capture phase segregation caused by gravity. Half of the fracture space surrounding the matrix columns occupy the domain $B_{\mathrm{f}}=\Omega_{\mathrm{f}} \times Z$. Furthermore, $B=\Omega \times Z$ denotes the small periodic units that occupy the domain $B=B_{\mathrm{f}} \cup B_{\mathrm{m}}$. All the small units together constitute the vertically fractured reservoir.

Fig. 1 a A vertically fractured network, b a matrix column, c a horizontal cross-section for a small unit that consists of the matrix part and fracture part

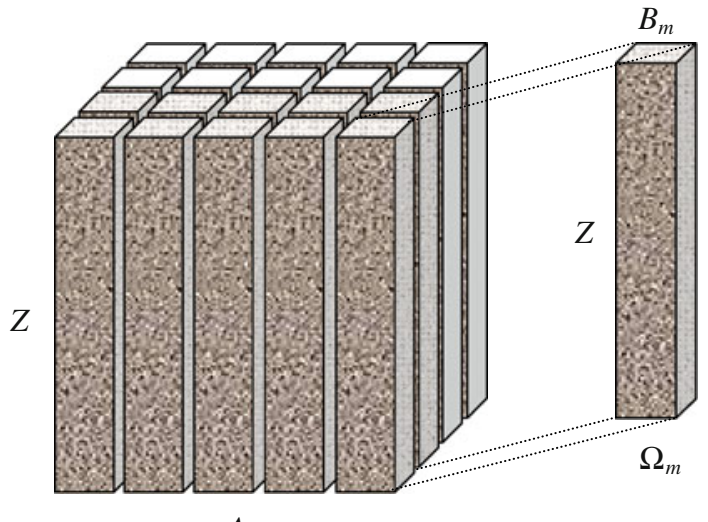

$A_{Q}$

(a)

(b)

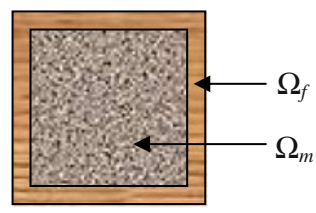

$\Omega$

(c) 
We simulate the injection of water into a vertically fractured oil reservoir. We apply continuity of capillary pressure and continuity of fluid flow as boundary conditions on the vertical interfaces between fracture and matrix columns. Flux continuity follows from fluid conservation at the interface between fracture and matrix. There is capillary pressure continuity at the boundary of fractures and matrix columns unless one of the phases either in the matrix or in fracture is immobile (Van Duijn et al. 1995). Indeed when one of the phases is immobile, the pressure of that phase depends on local conditions and cannot be determined globally. Hence, the capillary pressure, which is the difference between the phase pressure of the non-wetting phase and wetting phase, can be discontinuous. However, as residual saturations do not flow, this presents no difficulties for the modeling. Continuity of force, and hence continuity of phase pressures, implies continuity of capillary pressure when both phases are mobile.

Moreover, we incorporate the gravity effect directly inside the matrix columns. As a result, there is a net flow within the matrix columns. The symmetry of the fracture pattern in the horizontal plane is such that the fracture permeability can be considered isotropic. We only consider a two-phase (oil and water) incompressible flow where water viscosity, $\mu_{\mathrm{w}}$, and oil viscosity, $\mu_{\mathrm{o}}$, are assumed to be constant. As the main purpose of this article is to illustrate only the essential concepts, we assume that the fracture permeability is isotropic but this assumption can be easily relaxed (see below).

\subsection{Model Equations}

We use the two-phase ( $\alpha=0, w)$ extension of Darcy's law for constant fluid densities:

$$
\begin{gathered}
\mathbf{u}_{\alpha \mathrm{f}}^{*}=-\frac{k_{\mathrm{f}}^{*} k_{\mathrm{r} \alpha, \mathrm{f}}}{\mu_{\alpha \mathrm{f}}} \nabla\left(P_{\alpha \mathrm{f}}+\rho_{\alpha} g z\right):=-\lambda_{\alpha \mathrm{f}}^{*} \nabla \Phi_{\alpha \mathrm{f}}, \\
\mathbf{u}_{\alpha \mathrm{m}}=-\frac{k_{\mathrm{m}} k_{\mathrm{r} \alpha, \mathrm{m}}}{\mu_{\alpha \mathrm{m}}} \nabla \Phi_{\alpha \mathrm{m}}:=-\lambda_{\alpha \mathrm{m}} \nabla \Phi_{\alpha \mathrm{m}} .
\end{gathered}
$$

In these equations, the superscript $\left(^{*}\right)$ denotes the intrinsic (local) fracture properties. The intrinsic fracture permeability evaluated inside the fracture is denoted by $k_{\mathrm{f}}^{*}$. We define the intrinsic fracture permeability $k_{\mathrm{f}}^{*}$ based on the fracture aperture. The matrix permeability is denoted by $k_{\mathrm{m}}$. Relative permeabilities are denoted by $k_{\mathrm{rw}, \zeta}$ and $k_{\mathrm{ro}, \zeta}$, where $\zeta=\mathrm{f}$, m indicates the fracture or matrix systems. Here $P$ is the pressure, $\rho$ is the fluid density, $g$ is the gravity acceleration factor, and $z$ is the vertical upward direction. We define a phase mobility $\lambda_{\alpha}=k k_{\mathrm{r} \alpha} / \mu_{\alpha}$ as the ratio between the phase permeability and fluid viscosity. The phase potential $\Phi_{\alpha}$ is equal to the pressure plus the gravity term.

The mass conservation equation reads

$$
\frac{\partial}{\partial t}\left(\varphi_{\zeta} \rho_{\alpha \zeta} S_{\alpha \zeta}\right)+\nabla \cdot\left(\mathbf{u}_{\alpha \zeta} \rho_{\alpha \zeta}\right)=0
$$

where $\varphi$ is the porosity and $S_{\alpha}$ is the phase saturation. We obtain the governing equations describing incompressible two-phase flow by combining Darcy's Law (Eq. 1) and the mass conversation (Eq. 2)

$$
\frac{\partial}{\partial t}\left(\varphi_{\mathrm{f}}^{*} \rho_{\alpha \mathrm{f}} S_{\alpha \mathrm{f}}\right)=\nabla \cdot\left(\rho_{\alpha \mathrm{f}} \lambda_{\alpha \mathrm{f}}^{*} \nabla \Phi_{\alpha \mathrm{f}}\right) \quad \text { in } \Omega_{\mathrm{f}},
$$

and

$$
\frac{\partial}{\partial t}\left(\varphi_{\mathrm{m}} \rho_{\alpha \mathrm{m}} S_{\alpha \mathrm{m}}\right)=\nabla \cdot\left(\rho_{\alpha \mathrm{m}} \lambda_{\alpha \mathrm{m}} \nabla \Phi_{\alpha \mathrm{m}}\right) \quad \text { in } \Omega_{\mathrm{m}}
$$


We define a capillary pressure $P_{\mathrm{c}}=P_{\mathrm{o}}-P_{\mathrm{w}}$. At the interface between the fracture and matrix systems, there is continuity of water and oil flow

$$
\left(\rho_{\alpha \mathrm{f}} \lambda_{\alpha \mathrm{f}}^{*} \nabla \Phi_{\alpha \mathrm{f}}\right) \cdot \mathbf{n}=\left(\rho_{\alpha \mathrm{m}} \lambda_{\alpha \mathrm{m}} \nabla \Phi_{\alpha \mathrm{m}}\right) \cdot \mathbf{n} \text { on } \partial \Omega_{\mathrm{m}},
$$

where $\mathbf{n}$ denotes the outward unit normal vector to the surface $\left(\partial \Omega_{\mathrm{m}}\right)$ pointing from the matrix to the fracture. At the interface, there is also continuity of capillary pressure.

\section{Upscaling Technique}

We start with two-phase flow equations at the local scale to obtain the upscaled equations for a vertically fractured reservoir at the global scale using homogenization theory. The upscaling technique (homogenization) consists of five major steps.

The first step in the homogenization is the subdivision of the horizontal coordinates of the vertically fractured reservoir into two scales (see Fig. 1): local (small units) scale of size $(l)$ and the global scale of size $(L)$ that is much larger than the local scale. This implies that a condition for the application of homogenization is that separation of scales is possible. Our choice for the small unit (SU) scale is a single matrix column of which vertical faces are surrounded by fractures and its horizontal faces (e.g., top and bottom) are connected to the cap and base rock. We define $L$ as the dimension of the grid block scale. Note that each grid block consists of a few hundred small units. We define a scaling ratio $\varepsilon=l / L$ between the local scale and the global scale. A very large difference between the size of the global scale (grid block) and the local scale (SU) in addition to the low permeability of matrix columns, suggests that the oil flux from the matrix columns to fractures only leads to local scale variations of the fracture potential. As a consequence of the separation of scales, any space dependent quantity required to describe the physical process is a function of these two scales. Therefore, we can split the gradient operator $(\nabla)$ into a global scale (big) term, $\nabla_{\mathrm{b}}$, and a local scale (small) term, $\nabla_{\mathrm{s}}$, where $\nabla=\nabla_{\mathrm{b}}+\nabla_{\mathrm{s}}$. The matrix equation acts at the local scale. Thus, we do not apply the splitting procedure to the matrix domain. However, in a vertically fractured reservoir, there is no separation of scales in the vertical direction because the global scale is the same as the local scale. Therefore, we only apply the splitting procedure to the $x$ - and $y$-component of the fracture gradient operator. After applying this step to Eqs. 3-5, they read

$$
\begin{gathered}
\frac{\partial}{\partial t}\left(\varphi_{\mathrm{f}}^{*} S_{\alpha \mathrm{f}}\right)= \\
\nabla_{\mathrm{b}, x y} \cdot \lambda_{\alpha \mathrm{f}}^{*}\left(\nabla_{\mathrm{b}, x y} \Phi_{\alpha \mathrm{f}}+\nabla_{\mathrm{s}, x y} \Phi_{\alpha \mathrm{f}}\right) \\
+\nabla_{\mathrm{s}, x y} \cdot \lambda_{\alpha \mathrm{f}}^{*}\left(\nabla_{\mathrm{b}, x y} \Phi_{\alpha \mathrm{f}}+\nabla_{\mathrm{s}, x y} \Phi_{\alpha \mathrm{f}}\right) \\
+\nabla_{z} \cdot\left(\lambda_{\alpha \mathrm{f}}^{*} \nabla_{z} \Phi_{\alpha \mathrm{f}}\right) \quad \text { in } \Omega_{\mathrm{f}}, \\
\frac{\partial}{\partial t}\left(\varphi_{\mathrm{m}} S_{\alpha \mathrm{m}}\right)=\nabla_{\mathrm{s}, x y} \cdot\left(\lambda_{\alpha \mathrm{m}} \nabla_{\mathrm{s}, x y} \Phi_{\alpha \mathrm{m}}\right) \\
+\nabla_{z} \cdot\left(\lambda_{\alpha \mathrm{m}} \nabla_{z} \Phi_{\alpha \mathrm{m}}\right) \quad \text { in } \Omega_{\mathrm{m}}, \\
\left(\lambda_{\alpha \mathrm{f}}^{*}\left(\nabla_{\mathrm{b}, x y}+\nabla_{\mathrm{s}, x y}, \nabla_{z}\right) \Phi_{\alpha \mathrm{f}}\right) \cdot \mathbf{n}=\left(\lambda_{\alpha \mathrm{m}}\left(\nabla_{\mathrm{s}, x y}, \nabla_{z}\right) \Phi_{\alpha \mathrm{m}}\right) \cdot \mathbf{n} \quad \text { on } \partial \Omega_{\mathrm{m}} .
\end{gathered}
$$

Equations 3-5 have $\rho_{\alpha}$ as a common factor; therefore, it can be dropped in Eqs. 6-8.

The second step in the homogenization technique is writing the equations at the local scale in a dimensionless form using characteristic reference quantities. In our model, we use $l$ and $L$ as the characteristic lengths for the differentiation. This results in $\nabla_{\mathrm{D}}=\nabla_{\mathrm{b}}+\varepsilon^{-1} \nabla_{\mathrm{s}}$. The reference potential, $\Phi_{\mathrm{R}}$, is the potential difference between the injection and production well $(\Delta \Phi) \cdot t_{\mathrm{R}}=L^{2} \mu /(k \Delta \Phi)$ acts as the reference time for both the fracture and matrix systems. 
After non-dimensioning Eqs. 6-8, they reduce to

$$
\begin{aligned}
\frac{\partial}{\partial t}\left(\varphi_{\mathrm{f}}^{*} S_{\alpha \mathrm{f}}\right)= & \nabla_{\mathrm{b}, x y} \cdot \frac{k_{\mathrm{f}}^{*} k_{\mathrm{r} \alpha, \mathrm{f}}}{\mu_{\alpha \mathrm{f}}}\left(\nabla_{\mathrm{b}, x y} \Phi_{\alpha \mathrm{f}}+\frac{1}{\varepsilon} \nabla_{\mathrm{s}, x y} \Phi_{\alpha \mathrm{f}}\right) \\
& +\frac{1}{\varepsilon} \nabla_{\mathrm{s}, x y} \cdot \frac{k_{\mathrm{f}}^{*} k_{\mathrm{r} \alpha, \mathrm{f}}}{\mu_{\alpha \mathrm{f}}}\left(\nabla_{\mathrm{b}, x y} \Phi_{\alpha \mathrm{f}}+\frac{1}{\varepsilon} \nabla_{\mathrm{s}, x y} \Phi_{\alpha \mathrm{f}}\right) \\
& +\nabla_{z} \cdot\left(\frac{k_{\mathrm{f}}^{*} k_{\mathrm{r} \alpha, \mathrm{f}}}{\mu_{\alpha \mathrm{f}}} \nabla_{z} \Phi_{\alpha \mathrm{f}}\right) \text { in } \Omega_{\mathrm{f}},
\end{aligned}
$$

and

$$
\begin{gathered}
\frac{\partial}{\partial t}\left(\varphi_{\mathrm{m}} S_{\alpha \mathrm{m}}\right)= \\
\frac{1}{\varepsilon^{2}} \nabla_{\mathrm{s}, x y} \cdot\left(\lambda_{\alpha \mathrm{m}} \nabla_{\mathrm{s}, x y} \Phi_{\alpha \mathrm{m}}\right) \\
+\nabla_{z} \cdot\left(\lambda_{\alpha \mathrm{m}} \nabla_{z} \Phi_{\alpha \mathrm{m}}\right) \quad \text { in } \Omega_{\mathrm{m}} \\
\left(\lambda_{\alpha \mathrm{f}}^{*}\left(\nabla_{\mathrm{b}, x y}+\frac{1}{\varepsilon} \nabla_{\mathrm{s}, x y}, \nabla_{z}\right) \Phi_{\alpha \mathrm{f}}\right) \cdot \mathbf{n}=\left(\lambda_{\alpha \mathrm{m}}\left(\frac{1}{\varepsilon} \nabla_{\mathrm{s}, x y}, \nabla_{z}\right) \Phi_{\alpha \mathrm{m}}\right) \cdot \mathbf{n} \text { on } \partial \Omega_{\mathrm{m}} .
\end{gathered}
$$

The subscript D is dropped for reasons of concise notation. The non-dimensionalization procedure leads to some dimensionless numbers. Here, the dimensionless numbers are the permeability ratio and the scaling factor $(\varepsilon)$. We define the permeability ratio as the intrinsic (local) fracture permeability to the matrix permeability. We evaluate the permeability ratio with respect to the scaling ratio $\varepsilon$. Different upscaled equations are obtained when dimensionless numbers assume values of different orders of magnitude with respect to $\varepsilon$.

The third step in the homogenization technique is looking for the unknown quantities in the form of an asymptotic expansion in powers of $\varepsilon$. We expand the potential and phase saturation into contributions of decreasing significance with respect to $\varepsilon$

$$
\xi=\xi^{(0)}+\varepsilon \xi^{(1)}+\varepsilon^{2} \xi^{(2)}+\cdots,
$$

where $\xi$ denotes the potential or the saturation. Substituting these expansions into Eqs. 9-11 and collecting terms with the same powers of $\varepsilon$, we obtain partial differential equations recursively defining the entries in the asymptotic expansions.

The fourth step in the homogenization technique is solving the successive boundary value problems that are obtained after introducing the asymptotic expansion in the local dimensionless descriptions. We start by solving the equations of the lowest order in $\varepsilon$, i.e., the $1 / \varepsilon^{2}$ terms of Eq. 9 and the $1 / \varepsilon$ term of Eq. 11 . Using the fact that $k_{\mathrm{m}} / k_{\mathrm{f}}^{*} \approx \varepsilon^{2}$ leads to

$$
\nabla_{\mathrm{s}, x y} \cdot \frac{k_{\mathrm{f}}^{*} k_{\mathrm{r} \alpha, \mathrm{f}}^{(0)}}{\mu_{\alpha \mathrm{f}}} \nabla_{\mathrm{s}, x y} \Phi_{\alpha \mathrm{f}}^{(0)}=0 \quad \text { in } \Omega_{\mathrm{f}},
$$

and

$$
\frac{k_{\mathrm{f}}^{*} k_{\mathrm{r} \alpha, \mathrm{f}}^{(0)}}{\mu_{\alpha \mathrm{f}}} \nabla_{\mathrm{s}, x y} \Phi_{\alpha \mathrm{f}}^{(0)} \cdot \mathbf{n}=0 \quad \text { on } \partial \Omega_{\mathrm{m}} .
$$

Assuming periodic boundary conditions, the solution of this elliptic equation (phase potential) is independent of the local scale, i.e.,

$$
\Phi_{\alpha \mathrm{f}}^{(0)}=\Phi_{\alpha \mathrm{f}}^{(0)}\left(\mathbf{x}_{\mathrm{b}}, t\right) .
$$


It follows, if both phases are mobile, from the continuity of capillary pressure at the boundary between the fracture and the matrix that

$$
S_{\alpha \mathrm{f}}^{(0)}=S_{\alpha \mathrm{f}}^{(0)}\left(\mathbf{x}_{\mathrm{b}}, t\right) .
$$

Thus, the upscaled (macroscopic) fracture flow is defined on the global scale only.

Next, we consider the $1 / \varepsilon$ term of Eq. 9 and $\varepsilon^{0}$ of Eq. 11 . Using Eqs. 15 and 16 to cancel the local scale gradients of the independent terms of $\mathbf{x}_{\mathrm{s}}$ leads to

$$
\nabla_{\mathrm{s}, x y} \cdot \frac{k_{\mathrm{f}}^{*} k_{\mathrm{r} \alpha, \mathrm{f}}^{(0)}}{\mu_{\alpha \mathrm{f}}}\left(\nabla_{\mathrm{b}, x y} \Phi_{\alpha \mathrm{f}}^{(0)}+\nabla_{\mathrm{s}, x y} \Phi_{\alpha \mathrm{f}}^{(1)}\right)=0 \text { in } \Omega_{\mathrm{f}},
$$

and

$$
\left(\frac{k_{\mathrm{f}}^{*} k_{\alpha \mathrm{f}}^{(0)}}{\mu_{\alpha \mathrm{f}}} \nabla_{\mathrm{b}, x y} \Phi_{\alpha \mathrm{f}}^{(0)}+\frac{k_{\mathrm{f}}^{*} k_{\alpha \mathrm{f}}^{(0)}}{\mu_{\alpha \mathrm{f}}} \nabla_{\mathrm{s}, x y} \Phi_{\alpha \mathrm{f}}^{(1)}\right) \cdot \mathbf{n}=0 \quad \text { on } \partial \Omega_{\mathrm{m}} .
$$

In order to solve Eqs. 17 and 18, we assume that $\Phi^{(1)}$ is linearly related to $\nabla_{\mathrm{b}} \Phi_{\alpha \mathrm{f}}^{(0)}$, which in its most general form can be written as

$$
\Phi_{\alpha \mathrm{f}}^{(1)}=\left(\omega_{1}, \omega_{2}\right) \cdot \nabla_{\mathrm{b}, x y} \Phi_{\alpha \mathrm{f}}^{(0)},
$$

where the components $\omega_{1}$ and $\omega_{2}$ of the vector are the auxiliary functions at the local scale. We assume that $\omega_{1}$ and $\omega_{2}$ are independent of the global scale and $z$-coordinate. Substituting Eq. 19 in Eqs. 17 and 18 leads to

$$
\nabla_{\mathrm{s}, x y} \cdot \frac{k_{\mathrm{f}}^{*} k_{\mathrm{r} \alpha, \mathrm{f}}^{(0)}}{\mu_{\alpha \mathrm{f}}}\left(\left(\mathbf{I}+\nabla_{\mathrm{s}, x y} \otimes\left(\omega_{1}, \omega_{2}\right)\right) \cdot \nabla_{\mathrm{b}, x y} \Phi_{\alpha \mathrm{f}}^{(0)}\right)=0 \text { in } \Omega_{\mathrm{f}},
$$

and the boundary condition

$$
\left(\frac{k_{\mathrm{f}}^{*} k_{\mathrm{r} \alpha, \mathrm{f}}^{(0)}}{\mu_{\alpha \mathrm{f}}}\left(\left(\mathbf{I}+\nabla_{\mathrm{s}, x y} \otimes\left(\omega_{1}, \omega_{2}\right)\right) \cdot \nabla_{\mathrm{b}, x y} \Phi_{\alpha \mathrm{f}}^{(0)}\right)\right) \cdot \mathbf{n}=0 \quad \text { on } \partial \Omega_{\mathrm{m}} .
$$

The dyadic product $\nabla_{\mathrm{s}} \otimes \omega$ is a tensor with components $\partial \omega_{j} / \partial x_{i}$, where $i, j$ denote the $x$-, $y$-, $z$-coordinate. Equations 20 and 21 are equations for the local scale. Therefore, we need to eliminate $\nabla_{\mathrm{b}} \Phi_{\alpha \mathrm{f}}^{(0)}$ from Eqs. 20 and 21. We conclude from Eq. 15 that $\left(\partial / \partial x_{\mathrm{b}}, \partial / \partial y_{\mathrm{b}}\right) \Phi_{\alpha \mathrm{f}}^{(0)}$ can be approximated as a vector with constant components on the local scale (small unit). Therefore, Eqs. 20 and 21 reduce to

$$
\nabla_{\mathrm{s}, x y} \cdot \frac{k_{\mathrm{f}}^{*} k_{\mathrm{r} \alpha, \mathrm{f}}^{(0)}}{\mu_{\alpha \mathrm{f}}}\left(\nabla_{\mathrm{s}, x y} \otimes\left(\omega_{1}, \omega_{2}\right)\right)=0 \text { in } \Omega_{\mathrm{f}},
$$

and the boundary condition

$$
\frac{k_{\mathrm{f}}^{*} k_{\mathrm{r} \alpha, \mathrm{f}}^{(0)}}{\mu_{\alpha \mathrm{f}}}\left(\nabla_{\mathrm{s}, x y} \otimes\left(\omega_{1}, \omega_{2}\right)\right)=-\frac{k_{\mathrm{f}}^{*} k_{\mathrm{r} \alpha, \mathrm{f}}^{(0)}}{\mu_{\alpha \mathrm{f}}} \mathbf{I} \text { on } \partial \Omega_{\mathrm{m}} .
$$

Solving Eqs. 22 and 23 leads to the solutions of $\omega_{1}$ and $\omega_{2}$. These functions can be used to calculate the upscaled permeability tensor as shown below. By making various choices, we can obtain the components of $\omega$. For instance, we consider the longitudinal (flow) direction $\left(\partial / \partial x_{\mathrm{b}}, \partial / \partial y_{\mathrm{b}}\right) \Phi_{\alpha \mathrm{f}}^{(0)}=-\mathbf{e}_{x}$, where we use the minus sign because the potential decreases in the longitudinal direction. The $x$-component of the vector $\omega$ is denoted by $\omega_{1}=\omega \cdot \mathbf{e}_{x}$, and therefore $\omega \cdot \nabla_{\mathrm{b}, x y} \Phi_{\alpha \mathrm{f}}^{(0)}=-\omega_{1}$. We can interpret the $\omega_{1}$ as the first-order correction to 
the potential $\Phi^{(0)}$ when the system is subjected to a unit global gradient in the $x$-direction. The behavior of $\omega_{1}$ is therefore a measure of the potential fluctuation caused by the nonhomogeneous nature of a porous medium.

Finally, the third-order system $\left(\varepsilon^{0}\right.$ terms of Eq. 9 and $\varepsilon^{1}$ terms of Eq. 11) gives the upscaled model. The $\varepsilon^{0}$ terms of Eq. 9 are

$$
\begin{aligned}
\frac{\partial}{\partial t}\left(\varphi_{\mathrm{f}}^{*} S_{\alpha \mathrm{f}}^{(0)}\right)= & \nabla_{\mathrm{b}, x y} \cdot \frac{k_{\mathrm{f}}^{*} k_{\mathrm{r} \alpha, \mathrm{f}}^{(0)}}{\mu_{\alpha \mathrm{f}}}\left(\nabla_{\mathrm{b}, x y} \Phi_{\alpha \mathrm{f}}^{(0)}+\nabla_{\mathrm{s}, x y} \Phi_{\alpha \mathrm{f}}^{(1)}\right)+\nabla_{z} \cdot\left(\frac{k_{\mathrm{f}}^{*} k_{\mathrm{r} \alpha, \mathrm{f}}^{(0)}}{\mu_{\alpha \mathrm{f}}} \nabla_{z} \Phi_{\alpha \mathrm{f}}^{(0)}\right) \\
& +\nabla_{\mathrm{s}, x y} \cdot \frac{k_{\mathrm{f}}^{*} k_{\mathrm{r} \alpha, \mathrm{f}}^{(0)}}{\mu_{\alpha \mathrm{f}}}\left(\nabla_{\mathrm{b}, x y} \Phi_{\alpha \mathrm{f}}^{(1)}+\nabla_{\mathrm{s}, x y} \Phi_{\alpha \mathrm{f}}^{(2)}\right) \\
& +\nabla_{\mathrm{s}, x y} \cdot \frac{k_{\mathrm{f}}^{*} k_{\mathrm{r} \alpha, \mathrm{f}}^{(1)}}{\mu_{\alpha \mathrm{f}}}\left(\nabla_{\mathrm{b}, x y} \Phi_{\alpha \mathrm{f}}^{(0)}+\nabla_{\mathrm{s}, x y} \Phi_{\alpha \mathrm{f}}^{(1)}\right) \quad \text { in } \Omega_{\mathrm{f}} .
\end{aligned}
$$

The corresponding boundary condition with terms $\varepsilon^{1}$ reads

$$
\begin{gathered}
\left(\frac{k_{\mathrm{f}}^{*} k_{\mathrm{r} \alpha, \mathrm{f}}^{(0)}}{\mu_{\alpha \mathrm{f}}}\left(\nabla_{\mathrm{b}, x y} \Phi_{\alpha \mathrm{f}}^{(1)}+\nabla_{\mathrm{s}, x y} \Phi_{\alpha \mathrm{f}}^{(2)}\right)+\frac{k_{\mathrm{f}}^{*} k_{\mathrm{r} \alpha, \mathrm{f}}^{(1)}}{\mu_{\alpha \mathrm{f}}}\left(\nabla_{\mathrm{b}, x y} \Phi_{\alpha \mathrm{f}}^{(0)}+\nabla_{\mathrm{s}, x y} \Phi_{\alpha \mathrm{f}}^{(1)}\right)\right) \cdot \mathbf{n} \\
=\frac{1}{\varepsilon^{2}}\left(\frac{k_{\mathrm{m}} k_{\mathrm{r} \alpha, m}^{(0)}}{\mu_{\alpha \mathrm{m}}} \nabla_{\mathrm{s}, x y} \Phi_{\alpha \mathrm{m}}^{(0)}\right) \cdot \mathbf{n} \quad \text { on } \partial \Omega_{\mathrm{m}},
\end{gathered}
$$

where again we use the fact that $k_{\mathrm{m}} / k_{\mathrm{f}}^{*} \approx \varepsilon^{2}$.

The fifth step in the homogenization technique is obtaining the upscaled (macroscopic equivalent) model from Eqs. 24 and 25. We define the macroscopic average of any parameter q over the small unit $\Omega$ by integrating over $\Omega_{\mathrm{f}}$ and dividing by $|\Omega|$, i.e.,

$$
\langle\mathbf{q}\rangle=\frac{1}{|\Omega|} \int_{\Omega_{\mathrm{f}}} \mathbf{q} \mathrm{d} \mathbf{x}_{\mathrm{s}}
$$

In particular, considering the assumption of periodic local variations, the application of Gauss's theorem leads to

$$
\frac{1}{|\Omega|} \int_{\Omega_{\mathrm{f}}} \nabla_{\mathrm{s}} \cdot \mathbf{q} \mathrm{d} \mathbf{x}_{\mathrm{s}}=-\frac{1}{|\Omega|} \int_{\partial \Omega_{\mathrm{m}}} \mathbf{q} \cdot \mathbf{n} \mathrm{d} \sigma=-\frac{1}{|\Omega|} \int_{\Omega_{\mathrm{m}}} \nabla_{\mathrm{s}} \cdot \mathbf{q} \mathrm{d} \mathbf{x}_{\mathrm{s}} .
$$

In this equation, $\sigma$ denotes the coordinates of the boundary, $\partial \Omega_{\mathrm{m}}$. Note the fact that the normal vectors (n) changed direction when we go from $\Omega_{\mathrm{f}}$ to $\Omega_{\mathrm{m}}$.

We obtain the upscaled model by applying the macroscopic averaging procedure to Eq. 24 . To do so, we need to remove the local scale $\left(\partial / \partial_{\mathrm{S}}\right)$ dependence from Eq. 24 by applying the averaging procedure and Gauss's theorem to the boundary condition Eq. 25 and using Eq. 10. 
The third and fourth terms after the equal sign of Eq. 24 become

$$
\begin{aligned}
\frac{1}{|\Omega|} \int_{\Omega_{\mathrm{f}}}\left\{\nabla_{\mathrm{s}, x y} \cdot \frac{k_{\mathrm{f}}^{*} k_{\mathrm{r} \alpha, \mathrm{f}}^{(0)}}{\mu_{\alpha \mathrm{f}}}\left(\nabla_{\mathrm{b}, x y} \Phi_{\alpha \mathrm{f}}^{(1)}+\nabla_{\mathrm{s}, x y} \Phi_{\alpha \mathrm{f}}^{(2)}\right)\right. \\
\left.+\nabla_{\mathrm{s}, x y} \cdot \frac{k_{\mathrm{f}}^{*} k_{\mathrm{r} \alpha, \mathrm{f}}^{(1)}}{\mu_{\alpha \mathrm{f}}}\left(\nabla_{\mathrm{b}, x y} \Phi_{\alpha \mathrm{f}}^{(0)}+\nabla_{\mathrm{s}, x y} \Phi_{\alpha \mathrm{f}}^{(1)}\right)\right\} \mathrm{d} \mathbf{x}_{\mathrm{s}} \\
=-\frac{1}{|\Omega|} \iint_{\partial \Omega_{\mathrm{m}}}\left\{\nabla_{\mathrm{s}, x y} \cdot \frac{k_{\mathrm{f}}^{*} k_{\mathrm{r} \alpha, \mathrm{f}}^{(0)}}{\mu_{\alpha \mathrm{f}}}\left(\nabla_{\mathrm{b}, x y} \Phi_{\alpha \mathrm{f}}^{(1)}+\nabla_{\mathrm{s}, x y} \Phi_{\alpha \mathrm{f}}^{(2)}\right)\right. \\
\left.+\nabla_{\mathrm{s}, x y} \cdot \frac{k_{\mathrm{f}}^{*} k_{\mathrm{r} \alpha, \mathrm{f}}^{(1)}}{\mu_{\alpha \mathrm{f}}}\left(\nabla_{\mathrm{b}, x y} \Phi_{\alpha \mathrm{f}}^{(0)}+\nabla_{\mathrm{s}, x y} \Phi_{\alpha \mathrm{f}}^{(1)}\right)\right\} \cdot \mathbf{n d} \sigma \\
=-\frac{1}{|\Omega|} \int_{\partial \Omega_{\mathrm{m}}} \frac{1}{\varepsilon^{2}}\left(\frac{k_{\mathrm{m}} k_{\mathrm{r} \alpha, m}^{(0)}}{\mu_{\alpha \mathrm{m}}} \nabla_{\mathrm{s}, x y} \Phi_{\alpha \mathrm{m}}^{(0)}\right) \cdot \mathbf{n d} \sigma \\
=-\frac{1}{|\Omega|} \int_{\Omega_{\mathrm{m}}} \frac{1}{\varepsilon^{2}}\left\{\nabla_{\mathrm{s}, x y} \cdot\left(\frac{k_{\mathrm{m}} k_{\mathrm{r} \alpha, m}^{(0)}}{\mu_{\alpha \mathrm{m}}} \nabla_{\mathrm{s}, x y} \Phi_{\alpha \mathrm{m}}^{(0)}\right)\right\} \mathrm{d} \mathbf{x}_{\mathrm{s}} \\
=-\frac{1}{|\Omega|} \int\left\{\frac{\partial}{\partial t}\left(\varphi_{\mathrm{m}} S_{\alpha \mathrm{m}}^{(0)}\right)-\nabla_{z} \cdot\left(\lambda_{\alpha \mathrm{m}}^{(0)} \nabla_{z} \Phi_{\alpha \mathrm{m}}^{(0)}\right)\right\} \mathrm{d} \mathbf{x}_{\mathrm{s}} .
\end{aligned}
$$

This term is the exchange term of fluid flow at the interface between the fracture and matrix. In other words, it acts as an internal matrix source term in the upscaled fracture model, which means it shows how much water imbibes to the matrix and how much oil is fed to the fracture by the matrix columns.

The first and second terms after the equal sign of Eq. 24 can be written with Eq. 19 as

$$
\begin{aligned}
& \frac{1}{|\Omega|} \int_{\Omega_{\mathrm{f}}}\left\{\nabla_{\mathrm{b}, x y} \cdot \frac{k_{\mathrm{f}}^{*} k_{\mathrm{r} \alpha, \mathrm{f}}^{(0)}}{\mu_{\alpha \mathrm{f}}}\left(\nabla_{\mathrm{b}, x y} \Phi_{\alpha \mathrm{f}}^{(0)}+\nabla_{\mathrm{s}, x y} \Phi_{\alpha \mathrm{f}}^{(1)}\right)+\nabla_{z} \cdot\left(\frac{k_{\mathrm{f}}^{*} k_{\mathrm{r} \alpha, \mathrm{f}}^{(0)}}{\mu_{\alpha \mathrm{f}}} \nabla_{z} \Phi_{\alpha \mathrm{f}}^{(0)}\right)\right\} \mathrm{d} \mathbf{x}_{\mathrm{s}} \\
& =\frac{1}{|\Omega|} \int\left\{\nabla_{\Omega_{\mathrm{f}}, x y} \cdot\left(\frac{k_{\mathrm{f}}^{*} k_{\mathrm{r} \alpha, \mathrm{f}}^{(0)}}{\mu_{\alpha \mathrm{f}}}\left(\mathbf{I}+\nabla_{\mathrm{s}, x y} \otimes\left(\omega_{1}, \omega_{2}\right)\right) \cdot \nabla_{\mathrm{b}, x y} \Phi_{\alpha \mathrm{f}}^{(0)}\right)\right. \\
& \left.\quad+\nabla_{z} \cdot\left(\frac{k_{\mathrm{f}}^{*} k_{\mathrm{r} \alpha, \mathrm{f}}^{(0)}}{\mu_{\alpha \mathrm{f}}} \nabla_{z} \Phi_{\alpha \mathrm{f}}^{(0)}\right)\right\} \mathrm{d} \mathbf{x}_{\mathrm{s}} \\
& =\frac{1}{|\Omega|} \int\left\{\nabla_{\mathrm{b}} \cdot\left(\frac{k_{\mathrm{f}}^{*} k_{\mathrm{r} \alpha, \mathrm{f}}^{(0)}}{\mu_{\alpha \mathrm{f}}}\left(\mathbf{I}+\nabla_{\mathrm{s}} \otimes\left(\omega_{1}, \omega_{2}, 0\right)\right) \cdot \nabla_{\mathrm{b}} \Phi_{\alpha \mathrm{f}}^{(0)}\right)\right\} \mathrm{d} \mathbf{x}_{\mathrm{s}} \\
& =\nabla_{\mathrm{b}} \cdot\left(\frac{k_{\mathrm{f}} k_{\mathrm{r} \alpha, \mathrm{f}}^{(0)}}{\mu_{\alpha \mathrm{f}}} \nabla_{\mathrm{b}} \Phi_{\alpha \mathrm{f}}^{(0)}\right)
\end{aligned}
$$

where we define the effective fracture permeability tensor as follows:

$$
k_{\mathrm{f}}=\frac{1}{|\Omega|} \int_{\Omega_{\mathrm{f}}} k_{\mathrm{f}}^{*}\left(\mathbf{I}+\nabla_{\mathrm{s}} \otimes\left(\omega_{1}, \omega_{2}, 0\right)\right) \mathrm{d} \mathbf{x}_{\mathrm{s}} .
$$


Again, note that $\omega_{1}$ and $\omega_{2}$ are independent of the $z$-coordinate and the global scale. Douglas et al. (1993) have derived similar equations for special cases. Combining Eq. 24 with Eqs. 26 and 27 leads to the upscaled model for vertically fractured reservoirs

$$
\begin{aligned}
& \frac{\partial}{\partial t}\left(\varphi_{\mathrm{f}} S_{\alpha \mathrm{f}}^{(0)}\right)-\nabla_{\mathrm{b}} \cdot\left(\frac{k_{\mathrm{f}} k_{\mathrm{r} \alpha, \mathrm{f}}^{(0)}}{\mu_{\alpha \mathrm{f}}} \nabla_{\mathrm{b}} \Phi_{\alpha \mathrm{f}}^{(0)}\right) \\
& +\frac{1}{|\Omega|} \int_{\Omega_{\mathrm{m}}}\left\{\frac{\partial}{\partial t}\left(\varphi_{\mathrm{m}} S_{\alpha \mathrm{m}}^{(0)}\right)-\frac{\partial}{\partial z}\left(\lambda_{\alpha \mathrm{m}}^{(0)} \frac{\partial}{\partial z} \Phi_{\alpha \mathrm{m}}^{(0)}\right)\right\} \mathrm{d} \mathbf{x}_{\mathrm{s}}=0 \text { in } Q,
\end{aligned}
$$

where we derive the global fracture porosity as follows:

$$
\varphi_{\mathrm{f}}=\frac{1}{|\Omega|} \int_{\Omega_{\mathrm{f}}} \varphi_{\mathrm{f}}^{*} \mathrm{~d} \mathbf{x}_{\mathrm{s}} .
$$

The derivation of the upscaled model is complete. In the next section, we define characteristic dimensionless numbers.

\section{Dimensionless Numbers}

One of the advantages of homogenization is that it generates different upscaled models, when characteristic dimensionless numbers assume values of different orders of magnitude with respect to $\varepsilon$. In this case, where we are only dealing with the zeroth-order upscaled equation, we use the ratio between the fracture and matrix permeability as the characteristic dimensionless number. Other dimensionless numbers follow from the upscaled model equations. For these equations, we can additionally define the gravity number, i.e., gravity force over viscous force and the Peclet number. The Peclet number is defined as the ratio between the time required to imbibe water into the matrix column and the travel time of water in the fracture system. The relevant dimensionless numbers are discussed in a previous paper (Salimi and Bruining 2009), but for reasons of easy reference, we include the definition also in this article.

\subsection{Permeability Ratio in Fractures and Matrix}

The simplest definition of a fractured medium is a medium that contains fractures and matrix columns. From the fluid-flow point of view, such a medium is not necessarily a fractured medium, in particular when the matrix columns carry a substantial part of the flow. A fractured reservoir from the fluid-flow point of view means that the total volumetric flux $u_{\mathrm{f}}$ in fractures must be substantially larger than the total volumetric flux $u_{\mathrm{m}}$ in the matrix columns. It can be shown that the necessary requirement for this is that $k_{\mathrm{f}}^{*} \approx \varepsilon^{-2} k_{\mathrm{m}}$ because $\varepsilon$ is a measure of the fracture spacing. Indeed, flows in the fracture and matrix are subjected to approximately the same potential gradient. In addition, the fluid viscosities in the matrix and fracture are the same. Hence, the ratio between the velocity in the fracture and the matrix $u_{\mathrm{f}}^{*} / u_{\mathrm{m}}$ is of the order of magnitude $\approx \varepsilon^{-2}$. For a statistically homogeneous medium, the Darcy velocity is $u_{\mathrm{f}} \approx \varphi_{\mathrm{f}} u_{\mathrm{f}}^{*}$. Since in our case the global fracture porosity $\left(\varphi_{\mathrm{f}}\right)$ is of the order $\approx \varepsilon$, we obtain $u_{\mathrm{f}} / u_{\mathrm{m}} \approx \varepsilon^{-1}$. This can be expressed in terms of permeabilities, i.e., $k_{\mathrm{f}} / k_{\mathrm{m}} \approx \varepsilon^{-1}$, using Darcy's law. This means that if the matrix permeability is $10 \mathrm{mDarcy}$ and $\varepsilon \approx 0.01$, the intrinsic fracture permeability $k_{\mathrm{f}}^{*}$ must be of the order of $100 \mathrm{Darcy}$. The order of magnitude of the permeability ratio has also a consequence for a number of other aspects. If we assume that the capillary pressure is inversely proportional to the square root 
of the permeability $\sqrt{ } k$, it means that for the same saturation values, the capillary pressure in the matrix columns is 100 times as large as in the fracture.

\subsection{Peclet Number}

The Peclet number is defined here as the ratio between the time required to imbibe water into the matrix column and the travel time of water in the fracture system. The travel time of water in the fracture system is affected by imbibition in the matrix columns. For our model, we assume that counter-current imbibition in the matrix is the main recovery mechanism. Flow in the fractures is governed by multi-phase convection flows. Therefore, in this model, the Peclet number expresses the ratio between transport by convection (mainly viscous forces) in the fracture and the transport by capillary diffusion in the matrix. For other cases of interest, the relevant dimensionless number can be derived from the ratios of the residence time of the fluids in the matrix columns and fractures, respectively. For our situation, we derive the following expression for the Peclet number:

$$
P e=\frac{\ell^{2} u_{\mathrm{f}}}{D_{\text {cap }} L}, \quad \text { where } D_{\text {cap }}=-\frac{\lambda_{\mathrm{o}} \lambda_{\mathrm{w}}}{\lambda_{\mathrm{o}}+\lambda_{\mathrm{w}}} \frac{\mathrm{d} P_{\mathrm{c}}}{\mathrm{d} S_{\mathrm{w}}} .
$$

Here, $\lambda_{\alpha}$ is the mobility of phase $\alpha$ (oil, water), $l$ is the lateral matrix column size, and $L$ is the distance between wells. The qualitative behavior of water drive recovery in fractured media depends on the ratio of the characteristic time over which an amount of oil flows from the matrix to the fracture and the residence time of water in the fracture system.

If the residence time in the fracture system is small, it is expected that the recovery is controlled by the rate of imbibition from the matrix columns. In this case, most of the fractures contain mainly water. By capillary pressure continuity, this sets the boundary of the matrix columns at approximately zero capillary pressure.

If the residence time in the fracture system is large, water imbibes into the matrix columns before reaching the production well and releases an equal volume of oil (in the case of incompressible flow) to the fracture. In this case, there is a long period in which mainly oil is produced. After this period, water breakthrough occurs and the production performance depends on the detailed geometry of the fracture system and the well configurations. Therefore, from the fluid-flow point of view, the fractured reservoir behaves like a highly heterogeneous single-porosity reservoir rather than as a fractured reservoir.

\subsection{Gravity Number}

The gravity number is defined as the ratio between the gravity forces and the viscous forces. For the zeroth-order model, the periodic boundary condition implies that viscous forces are negligible with respect to gravity forces or capillary forces on the local scale. Therefore, we define the gravity number on the global (reservoir) scale, i.e., involving the characteristic length $L$ of the reservoir. The zeroth-order model follows from the most significant terms in the transport equations, i.e., those that scale with $\varepsilon^{0}$. We assert that this model is sufficiently accurate to grasp the essential features of flow in fractured reservoirs.

In view of what has been said above, we use the following expression for the gravity number (Yortsos 1991; Shook et al. 1992):

$$
N_{\mathrm{G}}=\frac{k_{\mathrm{f}} \Delta \rho g H}{\mu u_{\mathrm{f}} L},
$$


where $H$ is the height of the reservoir. As the Darcy velocity $u_{\mathrm{f}}$ is increasing, the viscous forces of the fracture system start to dominate gravity forces in the fracture system. On the other hand, when the injection velocity is small, gravity forces become dominant in the fracture system. This means that for this case, water tends to under-ride especially for high mobility ratios and gravity segregation happens.

\section{Numerical Solution}

The numerical procedure described below is an extension of the method used by Arbogast (1997) and Salimi and Bruining (2009) for the sugar cube model. The difficulty arises due to a coupling of flow in the vertical direction, which is important in the aggregated column model. From the computational point of view, we consider a matrix column associated with each point in the base plane of the reservoir. The horizontal cross-sectional position of any point $\mathbf{r}_{\mathrm{b}}=\left(x_{\mathrm{b}}, y_{\mathrm{b}}, z_{\mathrm{b}}\right) \in Q$, is denoted by $\mathbf{r}_{\mathrm{b}}^{\prime}=\left(x_{\mathrm{b}}, y_{\mathrm{b}}\right) \in A_{\mathrm{Q}}$. The matrix column at $\mathbf{r}_{\mathrm{b}}^{\prime}=\left(x_{\mathrm{b}}, y_{\mathrm{b}}\right) \in A_{\mathrm{Q}}$ is denoted by $B_{\mathrm{m}}\left(\mathbf{r}_{\mathrm{b}}^{\prime}\right)=\Omega_{\mathrm{m}}\left(\mathbf{r}_{\mathrm{b}}^{\prime}\right) \times H$, where this matrix column is representative of matrix columns in the vicinity of $\mathbf{r}^{\prime}$. We formulate our numerical method in terms of the water potential, the capillary pressure, and the water saturation. Here, we also define the capillary potential $\Phi_{\mathrm{c}}=P_{\mathrm{c}}+\left(\rho_{\mathrm{o}}-\rho_{\mathrm{W}}\right) g z$. We assume that all external sources, i.e., the production and injection wells, influence only the fractures. Equations 30 and 32 below are called the saturation equations, and the sums over the phases of each of the two-phase equations are 31 and 33, the pressure equations. Then, the upscaled equations in the fractured reservoir are

$$
\begin{aligned}
& \frac{\partial}{\partial t}\left(\varphi_{\mathrm{f}} S_{\mathrm{wf}}\right)-\nabla_{\mathrm{b}} \cdot\left(\lambda_{\mathrm{wf}} \nabla_{\mathrm{b}} \Phi_{\mathrm{wf}}\right) \\
& +\frac{1}{|\Omega|} \int_{\Omega_{\mathrm{m}}}\left\{\frac{\partial}{\partial t}\left(\varphi_{\mathrm{m}} S_{\mathrm{wm}}\right)-\frac{\partial}{\partial z}\left(\lambda_{\mathrm{wm}} \frac{\partial}{\partial z} \Phi_{\mathrm{wm}}\right)\right\} \mathrm{d} \mathbf{x}_{\mathrm{s}}=q_{\mathrm{ext}, \mathrm{w}} \quad \text { in } Q, \\
& -\nabla_{\mathrm{b}} \cdot\left(\left(\lambda_{\mathrm{wf}}+\lambda_{\mathrm{of}}\right) \nabla_{\mathrm{b}} \Phi_{\mathrm{wf}}+\lambda_{\mathrm{of}} \nabla_{\mathrm{b}} \Phi_{\mathrm{cf}}\right) \\
& +\frac{1}{|\Omega|} \int_{\Omega_{\mathrm{m}}}\left\{-\frac{\partial}{\partial z}\left(\left(\lambda_{\mathrm{wm}}+\lambda_{\mathrm{om}}\right) \frac{\partial}{\partial z} \Phi_{\mathrm{wm}}+\lambda_{\mathrm{om}} \frac{\partial}{\partial z} \Phi_{\mathrm{cm}}\right)\right\} \mathrm{d} \mathbf{x}_{\mathrm{s}}=q_{\mathrm{ext}, \mathrm{w}}+q_{\mathrm{ext}, \mathrm{o}} \text { in } Q
\end{aligned}
$$

where $q_{\text {ext,w }}$ and $q_{\text {ext,o }}$ are the external flow rates that come from the production and injection wells. The superscript (0) is dropped for reasons of concise notation. The equations on the matrix columns at $\mathbf{r}_{\mathrm{b}}^{\prime}$ are

$$
\begin{gathered}
\frac{\partial}{\partial t}\left(\varphi_{\mathrm{m}} S_{\mathrm{wm}}\right)-\nabla_{\mathrm{s}} \cdot\left(\lambda_{\mathrm{wm}} \nabla_{\mathrm{s}} \Phi_{\mathrm{wm}}\right)=0 \text { in } B_{\mathrm{m}}\left(r_{\mathrm{b}}^{\prime}\right) \\
-\nabla_{\mathrm{s}} \cdot\left(\left(\lambda_{\mathrm{wm}}+\lambda_{\mathrm{om}}\right) \nabla_{\mathrm{s}} \Phi_{\mathrm{wm}}+\lambda_{\mathrm{om}} \nabla_{\mathrm{s}} \Phi_{\mathrm{cm}}\right)=0 \text { in } B_{\mathrm{m}}\left(r_{\mathrm{b}}^{\prime}\right),
\end{gathered}
$$

The boundary conditions on the vertical faces of the matrix columns read

$$
\Phi_{\mathrm{wm}}\left(t, x_{\mathrm{b}}, x_{\mathrm{s}}\right)=\Phi_{\mathrm{wf}}\left(t, x_{\mathrm{b}}\right), \text { and } \Phi_{\mathrm{cm}}\left(t, x_{\mathrm{b}}, x_{\mathrm{s}}\right)=\Phi_{\mathrm{cf}}\left(t, x_{\mathrm{b}}\right) .
$$

There are no-flow boundary conditions on the top and bottom of the entire fractured reservoir, i.e.,

$$
-\lambda_{\alpha \zeta} \nabla \Phi_{\alpha \zeta} \cdot \mathbf{n}=0, \quad \alpha=\mathrm{w}, \mathrm{o}, \quad \text { and } \zeta=\mathrm{f}, \mathrm{m}
$$


Initially, there is capillary-gravity equilibrium both in the fracture system and in the matrix column. This means that the fluid exchange term between fracture and matrix is zero initially. Since initial oil in place is known, we determine the initial fracture water potential by solving the fracture pressure equation (Eq. 31). Due to equilibrium, we can solve the matrix pressure equation (Eq. 33) to obtain the initial matrix water potential.

Equations 30-35 cannot be solved sequentially or explicitly, because a small change in the boundary values on each matrix column can cause flow of a volume of fluid that is large in comparison to the volume of the fractures (Douglas et al. 1991). In other words, the matrix absorbs more fluid from the surrounding fractures in one-step than can be resident there. Part of the excess volume in the matrix is returned to the fractures in the next step, however, violating mass conservation. Therefore, the fracture-matrix interaction must be handled implicitly.

We use a backward Euler, time approximation for the complete system of Eqs. 30-35. We further use a fully implicit finite volume approach and first-order upwind scheme for spatial discretization. To facilitate the implementation of the no-flow boundary conditions and the continuity conditions of the potentials along the fracture-matrix interfaces, we discretize the space variables in a cell-centered manner in the fractures and also cell-centered with respect to $z$ in the matrix columns. However, the discretization in the $x_{\mathrm{s}}$ and $y_{\mathrm{s}}$ are vertex-centered. In this work, we use uniform grid cells in the fractures and in each matrix column. From the computational perspective, we consider a case in which the vertical discretization in the matrix columns coincides with that in the fractures.

We discretize $Q$ into $N_{\mathrm{xf}} \times N_{\mathrm{yf}} \times N_{\mathrm{zf}}$ grid cells, each grid cell of size $d_{\mathrm{xf}} \times d_{\mathrm{yf}} \times d_{\mathrm{zf}}$. The center of the fracture cell $\mathbf{p}=\left(p_{x}, p_{y}, p_{z}\right)$ is then

$$
\mathbf{x}_{\mathrm{bp}}=\left(\left(p_{x}-1 / 2\right) d_{\mathrm{xf}},\left(p_{y}-1 / 2\right) d_{\mathrm{yf}},\left(p_{z}-1 / 2\right) d_{\mathrm{zf}}\right),
$$

and the set of all fracture grid cell centers is

$$
N_{\mathrm{f}}=\left\{x_{\mathrm{bp}_{i}}: p_{i}=1,2,3, \ldots, N_{\mathrm{if}}, \quad i=x, y, z\right\} .
$$

This reduces the system of the equations to a fully discrete, three-dimensional problem. We denote the vectors of unknowns in the fracture by

$$
\begin{gathered}
\vec{\Phi}_{\mathrm{wf}}^{n}=\left\{\Phi_{\mathrm{wf}, i}^{n}, i=1,2,3, \ldots, N_{\mathrm{xf}} \times N_{\mathrm{yf}} \times N_{\mathrm{zf}}\right\}, \\
\vec{S}_{\mathrm{wf}}^{n}=\left\{S_{\mathrm{wf}, i}^{n}, i=1,2,3, \ldots, N_{\mathrm{xf}} \times N_{\mathrm{yf}} \times N_{\mathrm{zf}}\right\},
\end{gathered}
$$

where superscript $n$ denotes the time level $n$. In the vector, the potentials and saturations are stacked behind each other.

At each $\mathbf{x}_{\mathrm{bp}} \in N_{\mathrm{f}}$, there is a representative matrix column $B_{\mathrm{m}}\left(x_{\mathrm{bp}^{\prime}}\right)=\left(0, d_{x \mathrm{~m}} N_{x \mathrm{~m}}\right) \times$ $\left(0, d_{y \mathrm{~m}} N_{y \mathrm{~m}}\right) \times\left(0, d_{\mathrm{zf}} N_{\mathrm{zf}}\right)$, where $\mathbf{p}^{\prime}=\left(p_{x}, p_{y}\right)$ is the projection of $\mathbf{p}$ on the $x-y$ plane. Then, the center point of each matrix cell $\mathbf{c}=\left(c_{x}, c_{y}, c_{z}\right)$ is

$$
\mathbf{x}_{\mathrm{sp}^{\prime}}=\left(c_{x} d_{x \mathrm{~m}, \mathbf{p}^{\prime}}, c_{y} d_{y \mathrm{~m}, \mathbf{p}^{\prime}},\left(c_{z}-1 / 2\right) d_{\mathrm{xf}}\right)
$$

and the set of all matrix cell center points at fracture point $\mathbf{p}$ is given by

$$
N_{\mathrm{m}, \mathbf{p}^{\prime}}=\left\{x_{\mathrm{sp}^{\prime}, c_{i}}: c_{i}=0,1, \ldots, N_{i \mathrm{~m}, \mathbf{p}^{\prime}}, i=x, y ; c_{z}=1,2, \ldots, N_{\mathrm{zf}}\right\} .
$$

Then, associated with each grid point $i=1,2, \ldots, N_{\mathrm{xf}} \times N_{\mathrm{yf}} \times N_{\mathrm{zf}}$, we have two series of matrix unknowns

$$
\begin{aligned}
\vec{\Phi}_{\mathrm{wm}, i^{\prime}}^{n} & =\left\{\Phi_{\mathrm{wm}, i^{\prime} j}^{n}, i^{\prime}=1,2, \ldots, N_{\mathrm{xf}} \times N_{\mathrm{yf}}, j=1,2,3, \ldots, N_{x \mathrm{~m}} \times N_{y \mathrm{~m}} \times N_{\mathrm{zf}}\right\}, \\
\vec{S}_{\mathrm{wm}, i^{\prime}}^{n} & =\left\{S_{\mathrm{wm}, i^{\prime} j}^{n}, i^{\prime}=1,2, \ldots, N_{\mathrm{xf}} \times N_{\mathrm{yf}}, j=1,2,3, \ldots, N_{x \mathrm{~m}} \times N_{y \mathrm{~m}} \times N_{\mathrm{zf}}\right\},
\end{aligned}
$$


in the $m$ th matrix column. After that, we can write the fully discrete, nonlinear fracture and matrix equations

$$
\left\{\begin{array}{l}
F_{i}\left(\vec{\Phi}_{\mathrm{wf}}^{n}, \vec{S}_{\mathrm{wf}}^{n}, \vec{\Phi}_{\mathrm{wm}}^{n}, \vec{S}_{\mathrm{wm}}^{n}\right)=0, \quad i=1,2, \ldots, N_{\mathrm{xf}} \times N_{\mathrm{yf}} \times N_{\mathrm{zf}}, \\
M_{i^{\prime} j}\left(\vec{\Phi}_{\mathrm{wf}}^{n}, \vec{S}_{\mathrm{wf}}^{n}, \vec{\Phi}_{\mathrm{wm}, i^{\prime}}^{n}, \vec{S}_{\mathrm{wm}, i^{\prime}}^{n}\right)=0, \quad\left\{\begin{array}{c}
i^{\prime}=1,2, \ldots, N_{\mathrm{xf}} \times N_{\mathrm{yf}}, \\
j=1,2, \ldots, N_{x \mathrm{~m}} \times N_{y \mathrm{~m}} \times N_{\mathrm{zf}},
\end{array}\right.
\end{array}\right.
$$

where $F_{i}$ and $M_{i^{\prime} j}$ are some nonlinear functions. We use Newton's method to linearize the above system of equations. Let

$$
\vec{\Phi}_{\mathrm{wf}}^{n, k}, \vec{S}_{\mathrm{wf}}^{n, k}, \vec{\Phi}_{\mathrm{wm}}^{n, k}, \quad \text { and } \quad \vec{S}_{\mathrm{wm}}^{n, k}
$$

denote the $k$ th Newton iteration for the $n$th time level's solution. We write the evaluation of $F$ and $M$ at the $(k-1)$ th iteration for the $n$th time level solution as

$$
\left\{\begin{array}{l}
F_{i}^{n, k-1}=F_{i}\left(\vec{\Phi}_{\mathrm{wf}}^{n, k-1}, \vec{S}_{\mathrm{wf}}^{n, k-1}, \vec{\Phi}_{\mathrm{wm}}^{n, k-1}, \vec{S}_{\mathrm{wm}}^{n, k-1}\right), \\
M_{i^{\prime} j}^{n, k-1}=M_{i^{\prime} j}\left(\vec{\Phi}_{\mathrm{wf}}^{n, k-1}, \vec{S}_{\mathrm{wf}}^{n, k-1}, \vec{\Phi}_{\mathrm{wm}, i^{\prime}}^{n, k-1}, \vec{S}_{\mathrm{wm}, i^{\prime}}^{n, k-1}\right) .
\end{array}\right.
$$

Let $\partial_{\pi}$ denote the partial derivative with respect to $\pi$. We will develop an efficient numerical scheme based on the conventional Newton procedure. Such a conventional procedure would run as follows:

(1) Start with an initial guess for the solution

$$
\vec{\Phi}_{\mathrm{wf}}^{n, 0}, \vec{S}_{\mathrm{wf}}^{n, 0}, \vec{\Phi}_{\mathrm{wm}}^{n, 0} \text {, and } \vec{S}_{\mathrm{wm}}^{n, 0}
$$

Note that we use the initial water potential and water saturation as a first guess for the Newton iteration of the first time step. The initial capillary potentials $\Phi_{c}$ in the fracture system and in the matrix column should agree on the matrix column boundary, i.e., continuity of capillary pressure.

(2) For each $k=1,2, \ldots, n$ until convergence is reached:

(a) solve for

$$
\vec{\Phi}_{\mathrm{wf}}^{n, k}, \vec{S}_{\mathrm{wf}}^{n, k}, \vec{\Phi}_{\mathrm{wm}}^{n, k}, \quad \text { and } \quad \vec{S}_{\mathrm{wm}}^{n, k}
$$

satisfying

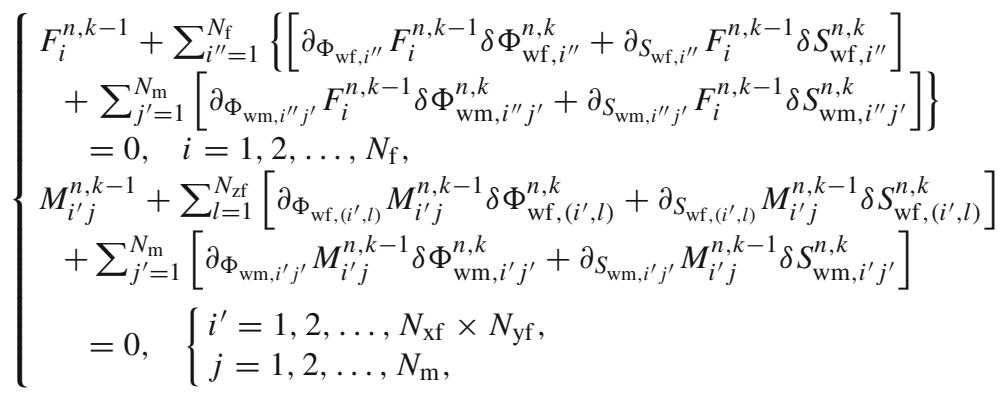

(b) update the potential and saturations

$$
\begin{array}{ll}
\vec{\Phi}_{\mathrm{wf}}^{n, k}=\vec{\Phi}_{\mathrm{wf}}^{n, k-1}+\delta \vec{\Phi}_{\mathrm{wf}}^{n, k}, & \vec{S}_{\mathrm{wf}}^{n, k}=\vec{S}_{\mathrm{wf}}^{n, k-1}+\delta \vec{S}_{\mathrm{wf}}^{n, k} \\
\vec{\Phi}_{\mathrm{wm}}^{n, k}=\vec{\Phi}_{\mathrm{wm}}^{n, k-1}+\delta \vec{\Phi}_{\mathrm{wm}}^{n, k}, & \vec{S}_{\mathrm{wm}}^{n, k}=\vec{S}_{\mathrm{wm}}^{n, k-1}+\delta \vec{S}_{\mathrm{wm}}^{n, k}
\end{array}
$$


This would complete the algorithm for a time step. The above linear system (Eq. 37) involves the solution of a $\left(2 \times\left(N_{\mathrm{f}}+N_{\mathrm{xf}} \times N_{\mathrm{yf}} \times N_{\mathrm{m}}\right)\right) \times\left(2 \times\left(N_{\mathrm{f}}+N_{\mathrm{xf}} \times N_{\mathrm{yf}} \times N_{\mathrm{m}}\right)\right)$ matrix for each Newton iteration at a time step, which is computationally expensive. Within the linearized Newton problem (Eq. 37), the matrix solutions in the $i$ th column depend on $\Phi_{\mathrm{wf},\left(i^{\prime}, l\right)}^{n, k}$ and $S_{\mathrm{wf},\left(i^{\prime}, l\right)}^{n, k}$, where $l=1,2, \ldots, N_{\mathrm{zf}}$. In other words, the matrix solution in the $i$ th column only depends on all the fracture cells surrounding the matrix column of interest. It is therefore possible to develop an efficient numerical scheme by decoupling the matrix and fracture problems without affecting the implicit nature of the scheme. We replace the matrix problem in Eq. 37 by the following three types of problems for

$$
\left(\tilde{\delta} \vec{\Phi}_{\mathrm{wm},\left(i^{\prime}, l\right)}^{n, m}, \tilde{\delta} \vec{S}_{\mathrm{wm},\left(i^{\prime}, l\right)}^{n, m}\right), \quad\left(\hat{\delta} \vec{\Phi}_{\mathrm{wm},\left(i^{\prime}, l\right)}^{n, m}, \hat{\delta} \vec{S}_{\mathrm{wm},\left(i^{\prime}, l\right)}^{n, m}\right), \quad \text { and } \quad\left(\bar{\delta} \vec{\Phi}_{\mathrm{wm}, i^{\prime}}^{n, m}, \bar{\delta} \vec{S}_{\mathrm{wm}, i^{\prime}}^{n, m}\right),
$$

where $\tilde{\delta}$ 's, $\hat{\delta}$ 's, and $\bar{\delta}$ 's satisfy three types of problems as follows:

First, for each $i^{\prime}=1,2, \ldots, N_{\mathrm{xf}} \times N_{\mathrm{yf}}$ and $j=1,2,3, \ldots, N_{\mathrm{m}}$,

$$
M_{i^{\prime} j}^{n, k-1}+\sum_{j^{\prime}=1}^{N_{\mathrm{m}}}\left[\partial_{\Phi_{\mathrm{wm}, i^{\prime} j^{\prime}}} M_{i^{\prime} j}^{n, k-1} \bar{\delta} \Phi_{\mathrm{wm}, i^{\prime} j^{\prime}}^{n, k}+\partial_{S_{\mathrm{wm}, i^{\prime} j^{\prime}}} M_{i^{\prime} j}^{n, k-1} \bar{\delta} S_{\mathrm{wm}, i^{\prime} j^{\prime}}^{n, k}\right]=0 .
$$

Second, for $l=1, \ldots, N_{\mathrm{zf}}$,

$\partial_{\Phi_{\mathrm{wf},\left(i^{\prime}, l\right)}} M_{i^{\prime} j}^{n, k-1}+\sum_{j^{\prime}=1}^{N_{\mathrm{m}}}\left[\partial_{\Phi_{\mathrm{wm}, i^{\prime} j^{\prime}}} M_{i^{\prime} j}^{n, k-1} \tilde{\delta} \Phi_{\mathrm{wm},\left(i^{\prime}, l\right), j^{\prime}}^{n, k}+\partial_{S_{\mathrm{wm}, i^{\prime} j^{\prime}}} M_{i^{\prime} j}^{n, k-1} \tilde{\delta} S_{\mathrm{wm},\left(i^{\prime}, l\right), j^{\prime}}^{n, k}\right]=0$.

Third, for $l=1, \ldots, N_{\mathrm{zf}}$,

$\partial_{S_{\mathrm{wf},\left(i^{\prime}, l\right)}} M_{i^{\prime} j}^{n, k-1}+\sum_{j^{\prime}=1}^{N_{\mathrm{m}}}\left[\partial_{\Phi_{\mathrm{wm}, i^{\prime} j^{\prime}}} M_{i^{\prime} j}^{n, k-1} \hat{\delta} \Phi_{\mathrm{wm},\left(i^{\prime}, l\right), j^{\prime}}^{n, k}+\partial_{S_{\mathrm{wm}, i^{\prime} j^{\prime}}} M_{i^{\prime} j}^{n, k-1} \hat{\delta} S_{\mathrm{wm},\left(i^{\prime}, l\right), j^{\prime}}^{n, k}\right]=0$.

If we multiply Eq. 39 by $\delta \Phi_{\mathrm{wf},\left(i^{\prime}, l\right)}^{n, k}$ and Eq. 40 by $\delta S_{\mathrm{wf},\left(i^{\prime}, l\right)}^{n, k}$ and then add these equations to Eq. 38, the result is identical to the matrix problem in Eq. 37. As a result, the matrix unknowns can be calculated by

$$
\delta \Phi_{\mathrm{wm}, i^{\prime} j}^{n, k}=\bar{\delta} \Phi_{\mathrm{wm}, i^{\prime} j}^{n, k}+\sum_{l=1}^{N_{\mathrm{zf}}}\left(\tilde{\delta} \Phi_{\mathrm{wm},\left(i^{\prime}, l\right), j}^{n, k} \delta \Phi_{\mathrm{wf},\left(i^{\prime}, l\right)}^{n, k}+\hat{\delta} \Phi_{\mathrm{wm},\left(i^{\prime}, l\right), j}^{n, k} \delta S_{\mathrm{wf},\left(i^{\prime}, l\right)}^{n, k}\right),
$$

and

$$
\delta S_{\mathrm{wm}, i^{\prime} j}^{n, k}=\bar{\delta} S_{\mathrm{wm}, i^{\prime} j}^{n, k}+\sum_{l=1}^{N_{\mathrm{zf}}}\left(\tilde{\delta} S_{\mathrm{wm},\left(i^{\prime}, l\right), j}^{n, k} \delta \Phi_{\mathrm{wf},\left(i^{\prime}, l\right)}^{n, k}+\hat{\delta} S_{\mathrm{wm},\left(i^{\prime}, l\right), j}^{n, k} \delta S_{\mathrm{wf},\left(i^{\prime}, l\right)}^{n, k}\right) .
$$

Equations 38-40 can be solved independently of the fracture system. Thus, we modify step 2(a) of the Newton Algorithm by first solving Eqs. 38-40. The changes in the fracture unknowns are then given by solving the fracture equations of 37, using implicitly definition of Eqs. 41 and 42. Subsequently, we explicitly use the changes in the fracture unknowns and Eqs. 41 and 42 to update the matrix $\delta$-potential and matrix $\delta$-saturation. Finally, the Newton iteration can be continued. This efficient numerical method is inexpensive as it only involves the solution of many $\left(2 \times N_{\mathrm{m}}\right) \times\left(2 \times N_{\mathrm{m}}\right)$ matrices and the solution of a $\left(2 \times N_{\mathrm{f}}\right) \times\left(2 \times N_{\mathrm{f}}\right)$ matrix as opposed to a single big matrix. 


\section{Results and Discussion}

In this section, we present the results of the numerical simulation. We consider a vertically fractured oil reservoir with a length of $500 \mathrm{~m}$, width of $200 \mathrm{~m}$, and height of $30 \mathrm{~m}$. Initially, the reservoir is saturated with oil. The water saturation both in the fracture and in the matrix is equal to the connate water saturations $\left(S_{\mathrm{wc}}\right)$. As shown in Fig. 2, water is injected through the entire height of the reservoir from one corner and subsequently oil and water are produced through the upper third of the reservoir height at the diagonally opposite corner. Table 1 shows the basic input data for the numerical simulations. For each simulation case, the water injection rate is uniform. Table 2 shows the calculated effective (global) fracture permeabilities based on the different values of the lateral matrix column size. Since fracture and matrix are considered as two different media, we use two different capillary pressure curves and relative permeability curves. Figures 3 and 4 show the capillary pressure curves and relative permeability curves both for the fracture and for the matrix. We discretize the fractured reservoir into $10 \times 5 \times 9$ grid cells in $x$-, $y$-, and $z$-direction. Hence, there are 450 grid cells for the fracture system, which contains $10 \times 5$ columns. Each column, which extends from the base rock to the cap rock, is subdivided in a stack of nine matrix blocks. Each matrix block contains $9 \times 9 \times 1$ grid cells in the $x-, y$-, and $z$-direction. In other words, corresponding to each fracture grid cell on the base plane, there is a single matrix column, consisting of a stack of nine matrix blocks. We use $9 \times 9 \times 9$ grid cells in $x$-, $y$-, and $z$-direction for each representative matrix column. Therefore, we have a total of $10 \times 5 \times 9$ grid cells for the fracture part and $(10 \times 5 \times 9) \times 9 \times 9$ grid cells to represent the matrix part leading to a total of 36,900 grid blocks. We examine the sensitivity of the numerical scheme by refining the discretization both in the fracture and in the matrix. Figure 5 shows the effect of fracture refinement on the cumulative oil production. As shown in Fig. 5, the numerical simulation is not sensitive to the fracture refinement as well as matrix refinement in the $z$-direction. However, Fig. 6 reveals that the choice of $9 \times 9 \times 9$ is slightly too coarse but still sufficiently accurate for a comparison between different cases. This is to be expected because the amount of oil in the fracture is small when compared to the amount of oil that resides in the matrix (Fractured reservoir of group A).

In the simulations, we vary the water injection rate, lateral matrix column size, and gravity. We also compare the upscaled aggregated column model with the upscaled sugar cube model (Salimi and Bruining 2009) for the same reservoir size and the same well configuration. Therefore, we investigate the effect of the water injection rate, lateral matrix column

Injection well perforated in

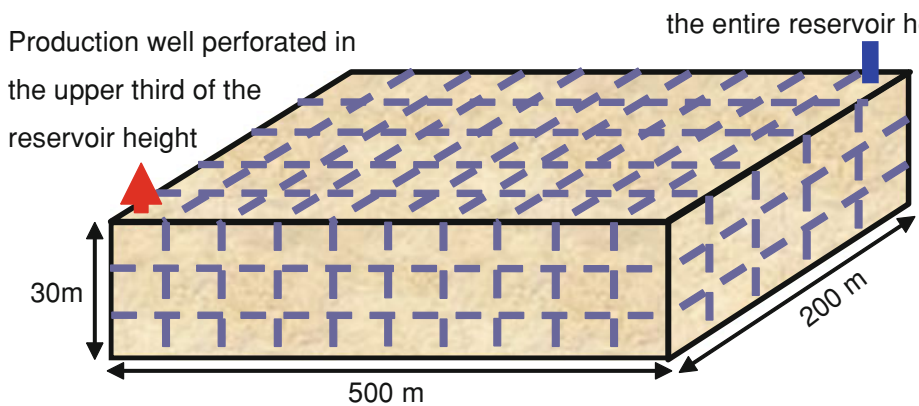

Fig. 2 Fractured reservoir waterflooding pattern 
Table 1 Data used in the numerical simulations

\begin{tabular}{llll}
\hline Initial reservoir pressure $(\mathrm{MPa})$ & 27.5 & Oil viscosity, $\mu_{\mathrm{o}}(\mathrm{Pas})$ & 0.002 \\
Bottom hole pressure for production wells $(\mathrm{MPa})$ & 26.9 & Oil density, $\rho_{\mathrm{O}}\left(\mathrm{kg} / \mathrm{m}^{3}\right)$ & 833 \\
Well radius $(\mathrm{m})$ & 0.1524 & Water viscosity, $\mu_{\mathrm{W}}(\mathrm{Pas})$ & 0.0005 \\
Fracture aperture $(\mu \mathrm{m})$ & 100 & Water density, $\rho_{\mathrm{W}}\left(\mathrm{kg} / \mathrm{m}^{3}\right)$ & 1,025 \\
Local fracture porosity, $\varphi_{\mathrm{f}}^{*}$ & 1 & Residual oil saturation in matrix & 0.3 \\
Intrinsic fracture permeability, $k_{\mathrm{f}}^{*}(\mathrm{D})$ & 844 & Residual oil saturation in fracture & 0 \\
Global fracture porosity based on $l=1 \mathrm{~m}, \varphi_{\mathrm{f}}$ & $2 \times 10^{-4}$ & Connate water saturation in matrix & 0.25 \\
Matrix porosity, $\varphi_{\mathrm{m}}$ & 0.19 & Connate water saturation in fracture & 0 \\
Matrix permeability, $k_{\mathrm{m}}(\mathrm{mD})$ & 1 & &
\end{tabular}

Table 2 Calculated effective (global) fracture permeability based on the lateral matrix column size

\begin{tabular}{lcc}
\hline $\begin{array}{l}\text { Lateral matrix column size, } l \\
(\mathrm{~m})\end{array}$ & $\begin{array}{l}\text { Effective fracture permeability in } \\
x \text { - and } y \text {-direction, } k_{\mathrm{f}, x y}(\mathrm{mD})\end{array}$ & $\begin{array}{l}\text { Effective fracture permeability in } \\
z \text {-direction, } k_{\mathrm{f}, z}(\mathrm{mD})\end{array}$ \\
\hline 0.5 & 166.67 & 333.33 \\
1 & 83.33 & 166.66 \\
2 & 41.67 & 83.33 \\
3 & 27.78 & 55.56 \\
4 & 20.83 & 41.66 \\
\hline
\end{tabular}

Fig. 3 Capillary pressure curves versus water saturation

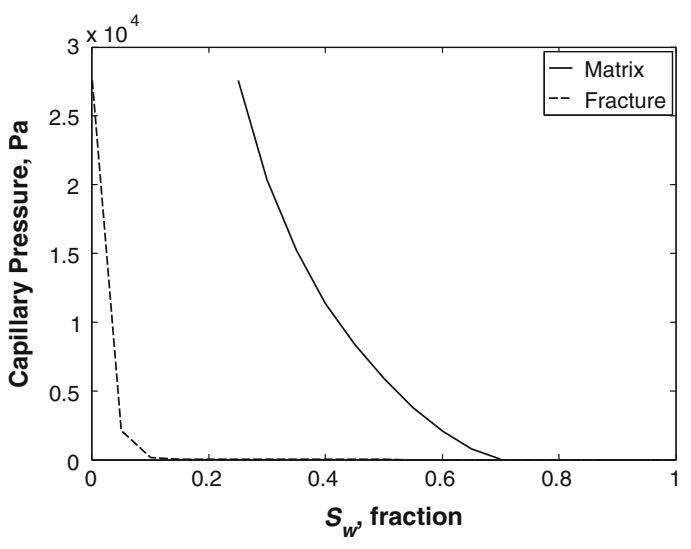

size, gravity, and the fluid-flow exchange term (aggregated column versus sugar cube). The dependence can be expressed in terms of the gravity number and the Peclet number. In all cases, the cumulative oil production is the basis for a comparison. Note that we express the (dimensionless) time in terms of cumulative PV water injected.

The results are organized as follows: first, we describe the effect of the lateral matrix column size for different water injection rates. Subsequently, we discuss the effect of the water injection rates on the cumulative oil production for different matrix column sizes. Third, we study the effect of gravity on the cumulative oil production, i.e., by performing the simulation at different gravity numbers. Next, we illustrate the effect of different reservoir geometries 
Fig. 4 Relative permeability curves versus water saturation

Fig. 5 Effect of fracture discretization on the cumulative oil production

Fig. 6 Effect of matrix discretization on the cumulative oil production
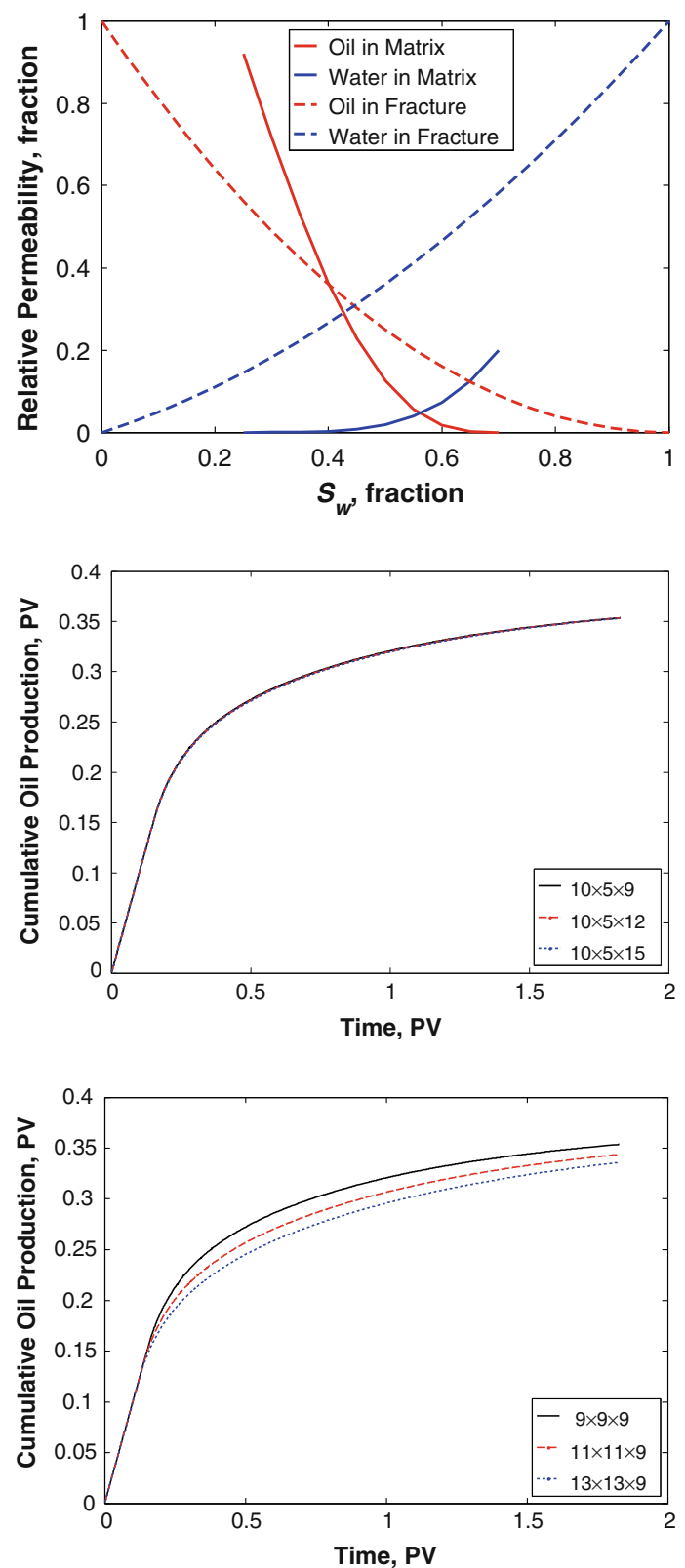

(i.e., aggregated column versus sugar cube), which lead to two different fluid-flow exchange terms in the upscaled models. After that, we define two different fluid-flow regimes by comparing the aggregated column model with the effective permeability model. Here, we use the Peclet number to distinguish different fluid-flow regimes. Finally, we investigate whether the aggregated column model can be solved with the current state of the art simulation programs, e.g., ECLIPSE simulations (i.e., the BWR approach) with a shape factor for which the vertical height is equal to the layer height. 

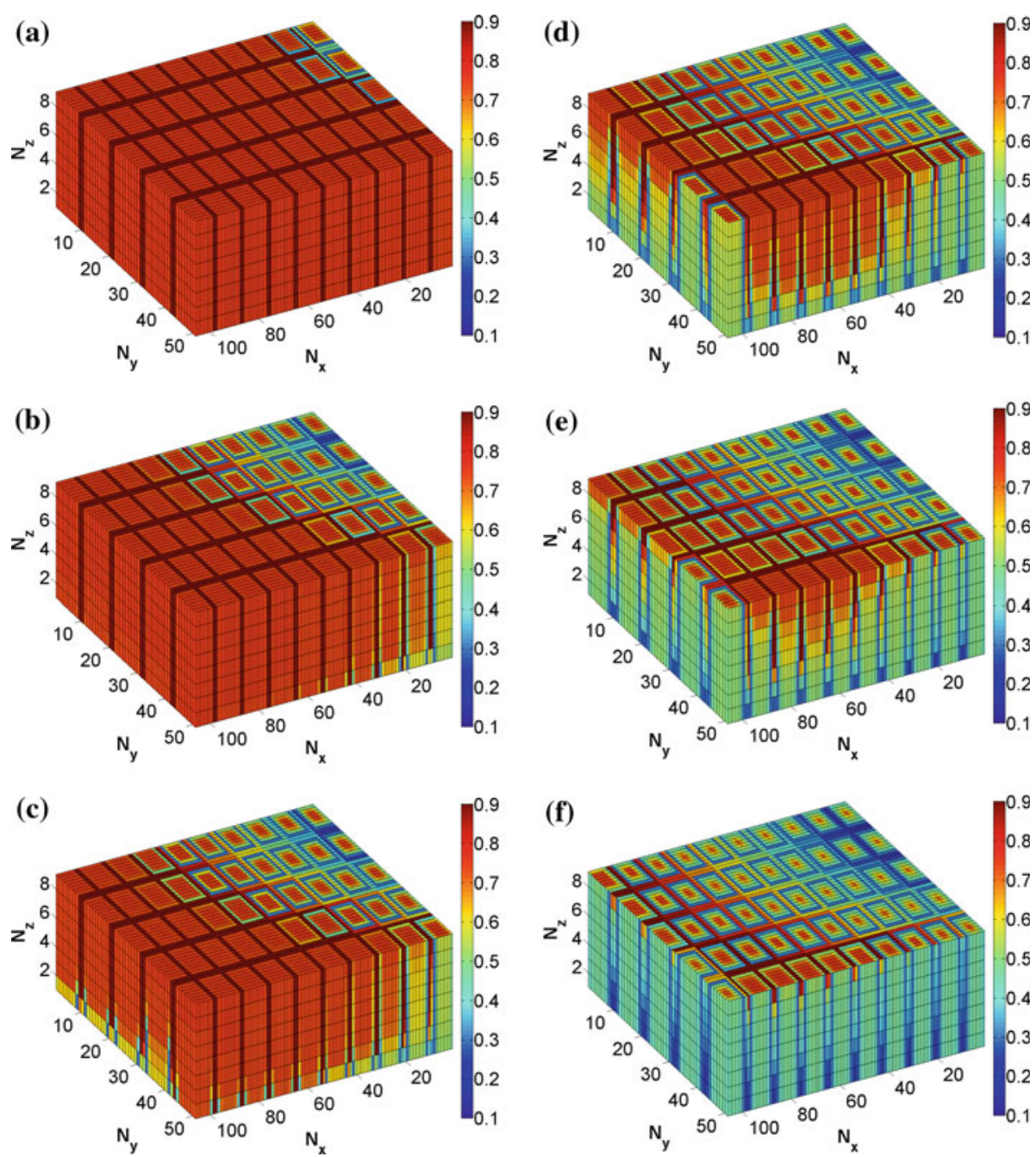

Fig. 7 Oil Saturation history, a time $=5$ days, b time $=37.5$ days, $\mathbf{c}$ time $=50$ days, $\mathbf{d}$ time $=75$ days, e time $=100$ days, and $\mathbf{f}$ time $=200$ days. The reservoir has a length of $500 \mathrm{~m}$ in the $x$-direction, $200 \mathrm{~m}$ in the $y$-direction and $30 \mathrm{~m}$ in the $z$-direction. The water injection rate is $1 \mathrm{PV}$ per year and the lateral matrix column size is $2 \mathrm{~m}$. First, the water occupies the bottom of the reservoir. Then, the water rises in the fractures and finally the water rises in the columns. Due to gravity, the oil at the top of the reservoir is not fully depleted

Figure 7 shows the oil saturation history after water injection in the two NE corner columns, with production in the top $1 / 3$ of the two SW corner columns. As opposed to the sugar cube model, the water saturation first expands via the bottom of the reservoir.

\subsection{Effect of the Lateral Matrix Column Size}

Figure $8 \mathrm{a}$ and $\mathrm{b}$ show the cumulative oil production for various lateral matrix column sizes at a water injection rate of $0.1 \mathrm{PV}$ per year and $10 \mathrm{PV}$ per year, respectively. We see from Fig. $8 \mathrm{a}$ and $\mathrm{b}$ that as the lateral matrix column size increases, the corresponding cumulative 
Fig. 8 a Effect of the lateral matrix column size on the cumulative oil production for a water injection rate of $0.1 \mathrm{PV}$ per year $\left(N_{\mathrm{G}} \approx 0.1\right)$, b Effect of the lateral matrix column size on the cumulative oil production for a water injection rate of $10 \mathrm{PV}$ per year $\left(N_{\mathrm{G}} \approx 10^{-4}\right)$
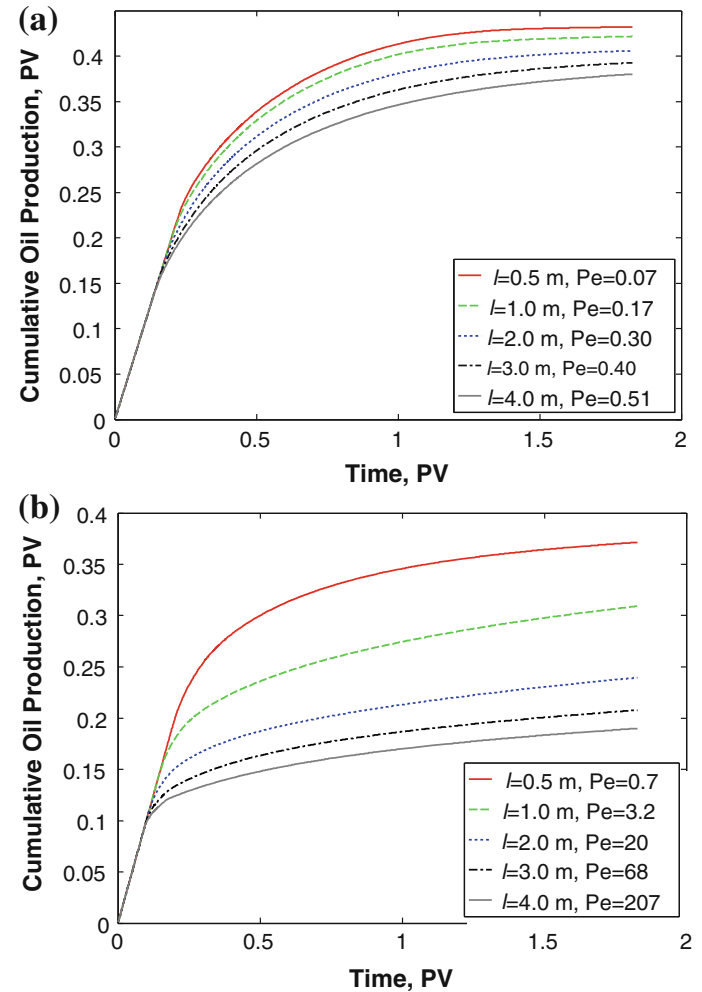

oil production decreases. This is to be expected because by increasing the lateral matrix columns size, the rate of counter-current imbibition in the matrix decreases. Therefore, the rate of oil and water exchange at the interface between fracture and matrix column reduces and consequently the Peclet number increases. As the rate of fluid-flow exchange acts as an internal source term in the upscaled equation, this leads to a lower oil recovery factor. We recall from the definition that the Peclet number is not proportional to the injection rate as it is influenced by imbibition in the matrix.

Figure $8 \mathrm{a}$ reveals that at a low water injection rate ( $0.1 \mathrm{PV}$ per year), the cumulative oil production for different lateral matrix column sizes has the same qualitative behavior in terms of oil production. Moreover, it shows that the order of magnitude of the Peclet number does not change by increasing the lateral matrix column size. When the water injection rate is low, the residence time in the fracture is long. Therefore, at these small Peclet numbers, even by reducing the rate of imbibition, there is enough time for water to imbibe into the matrix column before it reaches the production well. As a result, the cumulative oil production remains high.

On the other hand, Fig. $8 \mathrm{~b}$ shows that at a high water injection rate (10 PV per year) the cumulative oil production for different lateral column sizes changes significantly. Furthermore, the Peclet number also varies considerably by increasing the lateral matrix column size. This means that when the residence time for water in the fracture is small, the oil recovery mechanism is sensitive to the rate of oil and water exchange at the interface between fracture and matrix column. Even for these small residence times, a decreasing lateral matrix column 
size, which reduces the imbibition time into the matrix column, can lead to a larger delay in water breakthrough and thus a higher oil production.

\subsection{Effect of the Water Injection Rate}

Figure $9 \mathrm{a}$ and $\mathrm{b}$ show the cumulative oil production for a (square) lateral matrix column size of 0.5 and $4 \mathrm{~m}$ at different water injection rates. Note that we express the (dimensionless) time in terms of cumulative PV water injected. We observe from Fig. 9a and b that as the water injection rate increases, the corresponding cumulative oil production decreases. When the water injection rate increases, the rate of transport by convection in the fracture also increases meaning that the residence in the fracture decreases. Consequently, the Peclet number becomes larger and the oil recovery factor becomes lower.

As shown in Fig. 9a, the cumulative oil production for a small lateral matrix column size $(0.5 \mathrm{~m})$ at different water injection rates has the same qualitative behavior in terms of oil production. Figure 9a also reveals that the order of magnitude of the Peclet number does not change with increasing water injection rate. When the lateral matrix column size is small, the characteristic time of the water imbibition inside the matrix is short, which means that a major amount of oil in the matrix is depleted by water as soon as water reaches the boundary of the matrix column because of capillary pressure continuity. Moreover, the characteristic imbibition time is much shorter than the characteristic time of transport by convection in the fracture. Therefore, even by reducing the residence time in the fracture still the water imbibition in the matrix is dominant over convection in the fracture. As a result, increasing

Fig. 9 a Effect of the water injection rate on the cumulative oil production for a lateral matrix column size of $0.5 \mathrm{~m}$, b Effect of the water injection rate on the cumulative oil production for a lateral matrix column size of $4 \mathrm{~m}$
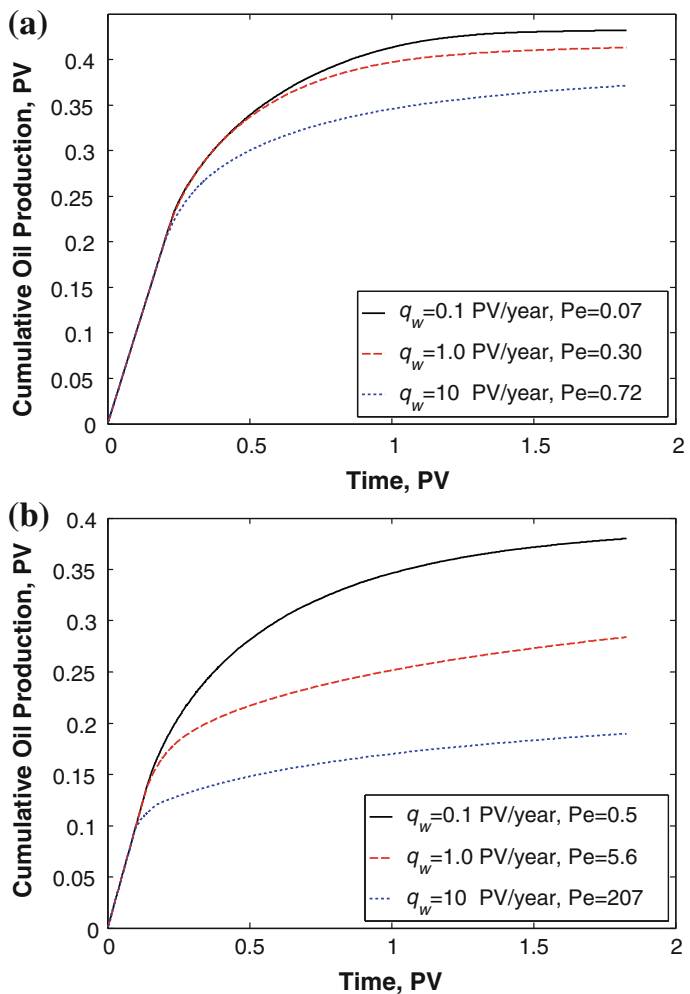
the water injection rate does not qualitatively influence the cumulative oil production for small lateral matrix column sizes (see Fig. 9a).

However, the cumulative oil production for a large lateral matrix column size $(4 \mathrm{~m})$ changes significantly by varying the water injection rate (Fig. 9b). Moreover, we see from Fig. 9b that the order of magnitude of the Peclet number also changes, which causes the different oil production behavior. Here, the time required for water to imbibe into the matrix is long because of the large lateral matrix column size $(4 \mathrm{~m})$, meaning that the characteristic time of water imbibition is long. Furthermore, at a water injection rate of $0.1 \mathrm{PV}$ per year the order of magnitude of imbibition in the matrix is almost the same as the characteristic time of convection in the fracture, i.e., $P e=0.5$. Therefore, at a higher water injection rate (i.e., $1 \mathrm{PV}$ per year and $10 \mathrm{PV}$ per year), the time required for transport by convection in the fracture becomes much shorter than the time needed for water to imbibe into the matrix column, i.e., $P e=5.63$ and $P e=207$. Consequently, this leads to a lower oil recovery factor.

Based on the results shown in Fig. 9a and b, we distinguish two different practical production scenarios. (1) If a fractured reservoir has a large heterogeneity in matrix column size (fracture spacing), the best strategy to have a high oil recovery factor is to use a low water injection rate. (2) If a fractured reservoir contains matrix columns with a relatively small size, the best strategy to have a high net present value as well as a relatively high oil recovery factor is to use high water injection rates.

\subsection{Effect of Gravity}

A comparison of the cumulative oil production for the aggregated column model with and without gravity is shown in Fig. 10a and b. For a small Peclet number and a large gravity number $\left(N_{\mathrm{G}}=0.1\right)$, it can be seen from Fig. 10a that at an early stage $(t<0.22 \mathrm{PV})$, using the aggregated column model both with gravity and without gravity, no major water breakthrough has yet occurred. For $0.22 \mathrm{PV}<t<1.5 \mathrm{PV}$, however, water breakthrough occurs earlier for the aggregated column model with gravity and hence leads to less oil production. Finally, the aggregated column model both with gravity and without gravity tend to the same value of the cumulative oil production. When gravity is included in the model, under-riding of water occurs, which causes earlier water breakthrough and hence leads initially to less oil production. Therefore, before water breakthrough $(t<0.22 \mathrm{PV})$ occurs in the presence of gravity, the results for both cases (i.e., with gravity and without gravity) are the same. After that, water breaks through in the aggregated column model with gravity while the aggregated column model without gravity produces mainly oil $(0.22 \mathrm{PV}<t<0.8 \mathrm{PV})$. Subsequently, water breakthrough also occurs after almost all mobile oil has been produced. However, the water level in a case where gravity is included rises gradually because the top layer in the production well is perforated (see, however, Fig. 7). Consequently, water imbibes in the top parts of the matrix columns, leading to oil production. Hence, for long times, the aggregated column model with gravity predicts almost the same cumulative oil production as obtained without gravity.

Figure 10b reveals that for much higher Peclet numbers and much smaller gravity numbers $\left(N_{\mathrm{G}}=9.9 \times 10^{-4}\right)$, the results of the aggregated column model with and without gravity almost coincide during the entire production period. Even as water never reaches the top layer of the fractures when gravity is included, it does not influence the cumulative oil production because the amount of oil in the fracture is small. Here, the small effect of gravity can be attributed to the fact that both capillary imbibition forces and viscous forces act against gravity forces. The capillary imbibition forces are constant for every case considered here. Moreover, gravity forces are also constant because the size of the reservoirs and 
Fig. 10 a Effect of gravity on the cumulative oil production for a water injection rate of $0.1 \mathrm{PV}$ per year and for a lateral matrix column size of $0.5 \mathrm{~m}$, b Effect of gravity on the cumulative oil production for a water injection rate of $1 \mathrm{PV}$ per year and for a lateral matrix column size of $4 \mathrm{~m}$
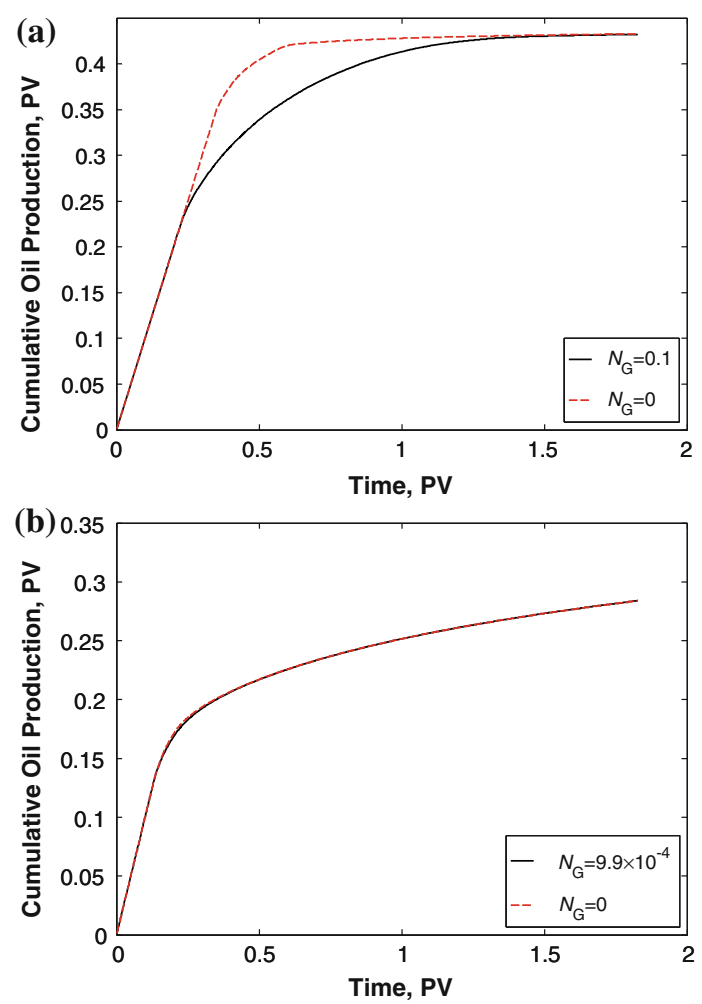

the density difference between oil and water are kept constant. The viscous forces change on the global scale because we vary the rate of water injection as well as the lateral matrix column size. When the Peclet number is small and gravity number is large, the viscous forces are small and therefore the effects of gravity forces can be observed. On the other hand, at a high Peclet number and very small gravity number, the viscous forces are at least one order of magnitude larger than gravity forces. Hence, the viscous forces become dominant and considering gravity forces for these cases does not have a large impact on the cumulative oil production. This result is very sensitive to the well outlay and other geometrical conditions.

\subsection{Effect of the Fractured Reservoir Topology}

Figure 11a and $\mathrm{b}$ show the difference in cumulative oil production for the aggregated column model and the sugar cube model. Figure 11a shows the result at a low Peclet number. In this case, the sugar cube model and the aggregated column model lead to almost the same result. However, for high Peclet numbers, the sugar cube model (see Fig. 11b) shows a much higher recovery than the aggregated column model. The reason is that at high Peclet numbers, the imbibition over the full column height is slow and hence oil production from the top of the columns is small. 
Fig. 11 a Effect of the fractured reservoir topology, i.e., aggregated column versus sugar cube model, for a water injection rate of $0.1 \mathrm{PV}$ per year and for a lateral matrix column size of $0.5 \mathrm{~m}\left(P e=0.07, N_{\mathrm{G}}=0.1\right)$,

b Effect of the fractured reservoir topology, i.e., aggregated vertical column versus sugar cube model, for a water injection rate of $10 \mathrm{PV}$ per year and for a lateral matrix column size of $4 \mathrm{~m}(\mathrm{Pe}=207$, $N_{\mathrm{G}}=10^{-4}$ )
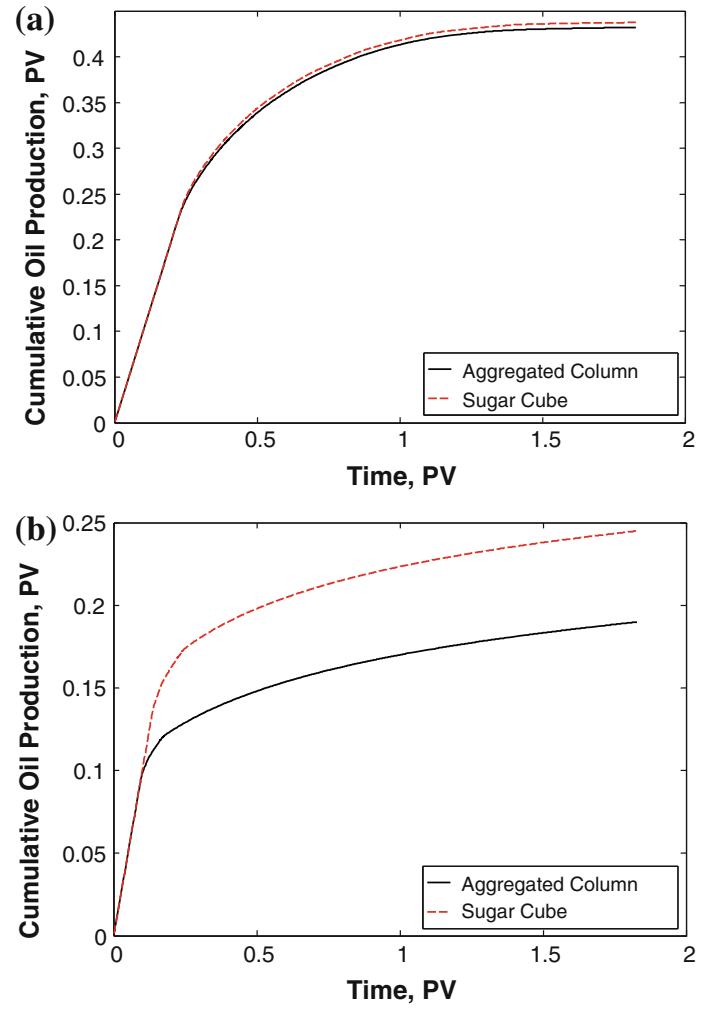

\subsection{Comparison Between the Aggregated Column Model and the Effective Permeability Model}

Figure 12a and $\mathrm{b}$ show a comparison of the cumulative oil production for the aggregated column model with the effective permeability model. For a low Peclet number (0.07) (see Fig. 12a), we observe that the aggregated column model predicts a smaller oil production than the effective permeability model when more than $0.22 \mathrm{PV}$ of water is injected. Clearly for incompressible fluids, the oil production rate and water injection rate before breakthrough are the same, i.e., for $t<0.22 \mathrm{PV}$. The fracture volume is $0.001 \mathrm{PV}$, meaning that the oil is mainly produced from the matrix. After $t>1 \mathrm{PV}$, the results of the aggregated column model and effective permeability model almost coincide. However, at higher Peclet numbers (e.g., 207 in Fig. 12b), the discrepancy between the aggregated column model and the effective permeability model significantly increases, where the aggregated column model predicts an oil recovery factor that is almost two times smaller than the oil recovery factor estimated by the effective permeability model.

If the Peclet number is small, the rate of oil and water exchange at the interface between matrix column and fracture is fast compared to the rate of convection in the fractures. In this case, the residence time in the fracture system is large and most water imbibes into the matrix columns and releases an equal volume of oil to the fracture. Therefore, there is a long period when mainly oil is produced at a rate equal to the injection rate (Fig. 12a). As a result, considering a precise rate of fluid exchange at the interface between matrix and fracture is not 
Fig. 12 a A comparison of the cumulative oil production for the aggregated column model with the effective permeability model for a water injection rate of $0.1 \mathrm{PV}$ per year and for a lateral matrix column size of $0.5 \mathrm{~m}$ $\left(P e=0.07, N_{\mathrm{G}}=0.1\right), \mathbf{b} \mathrm{A}$ comparison of the cumulative oil production for the aggregated column model with the effective permeability model for a water injection rate of $10 \mathrm{PV}$ per year and for a lateral matrix column size of $4 \mathrm{~m}\left(P e=207, N_{\mathrm{G}}=10^{-4}\right)$
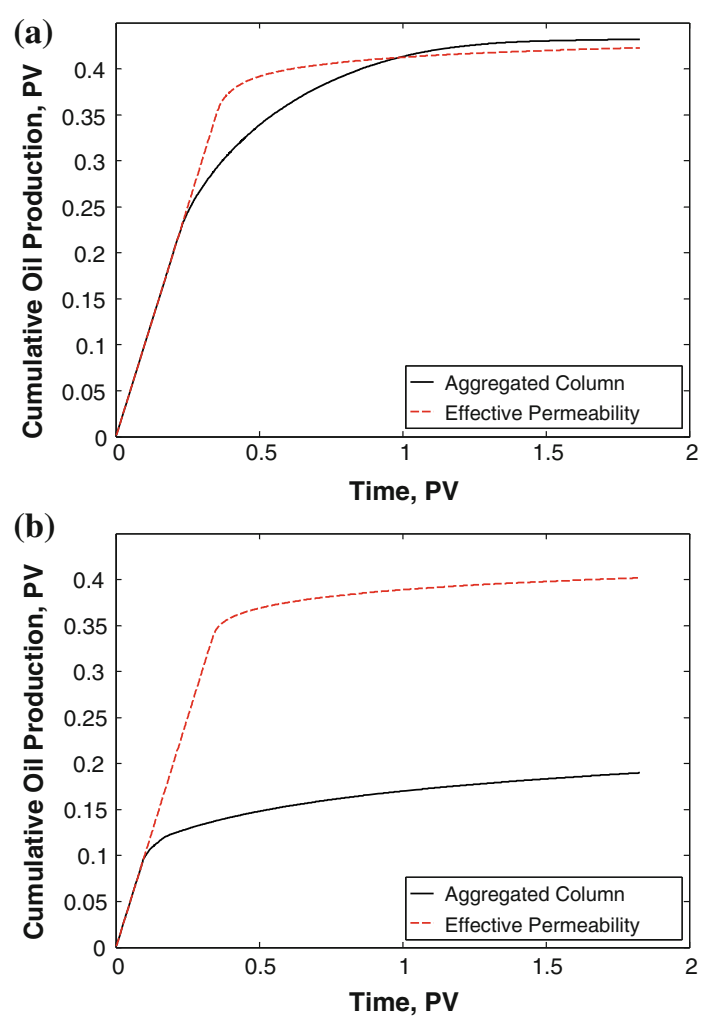

required for the prediction of oil recovery from waterflooded vertically fractured reservoirs. For this reason, one can use the effective permeability model (see Fig. 12a) instead of using the aggregated column model. Note that for the effective permeability model, we solved the equations, which correspond to a single conventional porosity model, with porosity equal to the matrix porosity and permeability equal to the effective permeability obtained from homogenization. Therefore, the computation time of the effective permeability model is a few hundred times smaller than that of the homogenized model.

On the other hand, if the Peclet number is large, the rate of oil and water exchange at the interface between the matrix column and the fracture is small compared to the rate of convection in the fractures. Consequently, the residence time in the fracture system is small. Therefore, it is expected that the recovery is controlled by the rate of counter-current imbibition from the matrix columns. Hence, one must use the aggregated column model to improve oil recovery predictions from a waterflooded vertically fractured reservoir rather than using the effective permeability model. In this case, most of the fractures contain mainly water. By capillary continuity, this situation sets the boundary of the matrix columns at the approximately zero capillary pressure or a water saturation of $\left(1-S_{\text {or }}\right)$. As a result of this, there is a short period in which mainly oil is produced (Fig. 12b), after which oil production becomes very slow. 


\subsection{Comparison Between the Aggregated Column Model and ECLIPSE Simulator (BWR Approach)}

Figure 13a-c show a comparison of the cumulative oil and water production for the aggregated column model with the ECLIPSE simulator. For a small Peclet number (0.07, Fig. 13a), the model and the simulator show the same breakthrough time, i.e., $t=0.22 \mathrm{PV}$. Subsequently, at $0.22 \mathrm{PV}<t<0.67 \mathrm{PV}$, the ECLIPSE simulator predicts a higher oil production rate than the aggregated column model as illustrated by the fact that the slope of cumulative oil production curve for the ECLIPSE simulator at this stage is steeper than the slope of the cumulative oil production curve for the aggregated column model. Finally, both the aggregated column model and ECLIPSE results tend to different values of oil production. The discrepancy in Fig. 13a between the aggregated column model and ECLIPSE is due to large gravity effects, which requires an accurate representation of the fracture-matrix interaction. For a slightly higher Peclet number (Fig. 13b), we see that the cumulative oil production for the aggregated column model and the ECLIPSE simulator almost coincide. Therefore, in this condition, the aggregated column model can be replaced by the BWR approach without appreciable loss of accuracy.

The results at still higher Peclet numbers are shown in Fig. 13c. In this case, the aggregated column model predicts a higher oil production at an early stage than the ECLIPSE simulator because of fast depletion of oil from the part of the matrix column adjacent to the fracture resulting from continuity of capillary pressure. Afterwards, the predicted rate of oil production of ECLIPSE exceeds the rate of oil production of the aggregated column model, and gradually the cumulative oil production from the ECLIPSE simulator reaches the value of the cumulative oil production for the aggregated column model.

The most important reason for the discrepancy between the aggregated column model and ECLIPSE model is the difference between the BWR transfer function and the fluidflow exchange term based on homogenization. The second most important reason is that three-dimensional matrix-block subgridding to the best of our knowledge is not available in the ECLIPSE simulator. The third most important reason is that there is no option in the ECLIPSE simulator for the aggregated column topology. We observe that most of the discrepancy between the aggregated column model and ECLIPSE simulator happens at higher and lower Peclet numbers. In these cases, accurate fluid-flow exchange terms are necessary for the accurate prediction of oil recovery because the oil recovery is controlled by the rate of imbibition for high Peclet numbers and by gravity for high gravity numbers. Consequently, satisfying the continuity of the capillary pressure has a significant effect on the cumulative oil production.

Figures $12 \mathrm{a}$ and $13 \mathrm{~b}$ show a small difference between the aggregated column model and the effective permeability model and ECLIPSE. We explain this observation as follows. When the Peclet number is small but not so small that gravity starts to dominate (see Fig. 13a), the rate of fluid transport in the fracture controls the oil recovery mechanism. Therefore, in this regime, considering an accurate rate of fluid exchange at the interface between matrix and fracture is not required for the prediction of oil recovery from waterflooded vertically fractured reservoirs. For this reason, one can use either the effective permeability model or the BWR approach (see Fig. 13b) instead of using the aggregated column model. Hence, when the Peclet number is small, but not so small that gravity starts to dominate, we can use either the effective permeability model or the BWR approach (Fig. 13b), instead of using the aggregated column model. Note that the effective permeability model does not have an exchange term because it is a single-porosity model. Therefore, it can be easily implemented and uses little computational time compared to the other two models. On the other hand, 
Fig. 13 a A comparison of the cumulative oil production between the aggregated column model and ECLIPSE simulator (BWR approach) for a water injection rate of $0.1 \mathrm{PV}$ per year and for a lateral matrix column size of $0.5 \mathrm{~m}(\mathrm{Pe}=0.07$, $N_{\mathrm{G}}=0.1$ ), b A comparison of the cumulative oil production between the aggregated column model and ECLIPSE simulator (BWR approach) for a water injection rate of $1 \mathrm{PV}$ per year and for a lateral matrix column size of $1 \mathrm{~m}\left(P e=0.45, N_{\mathrm{G}}=0.005\right)$, c A comparison of the cumulative oil production between the aggregated column model and ECLIPSE simulator (BWR approach) for a water injection rate of $1 \mathrm{PV}$ per year and for a lateral matrix column size of $2 \mathrm{~m}$ $\left(P e=1.01, N_{\mathrm{G}}=0.002\right)$
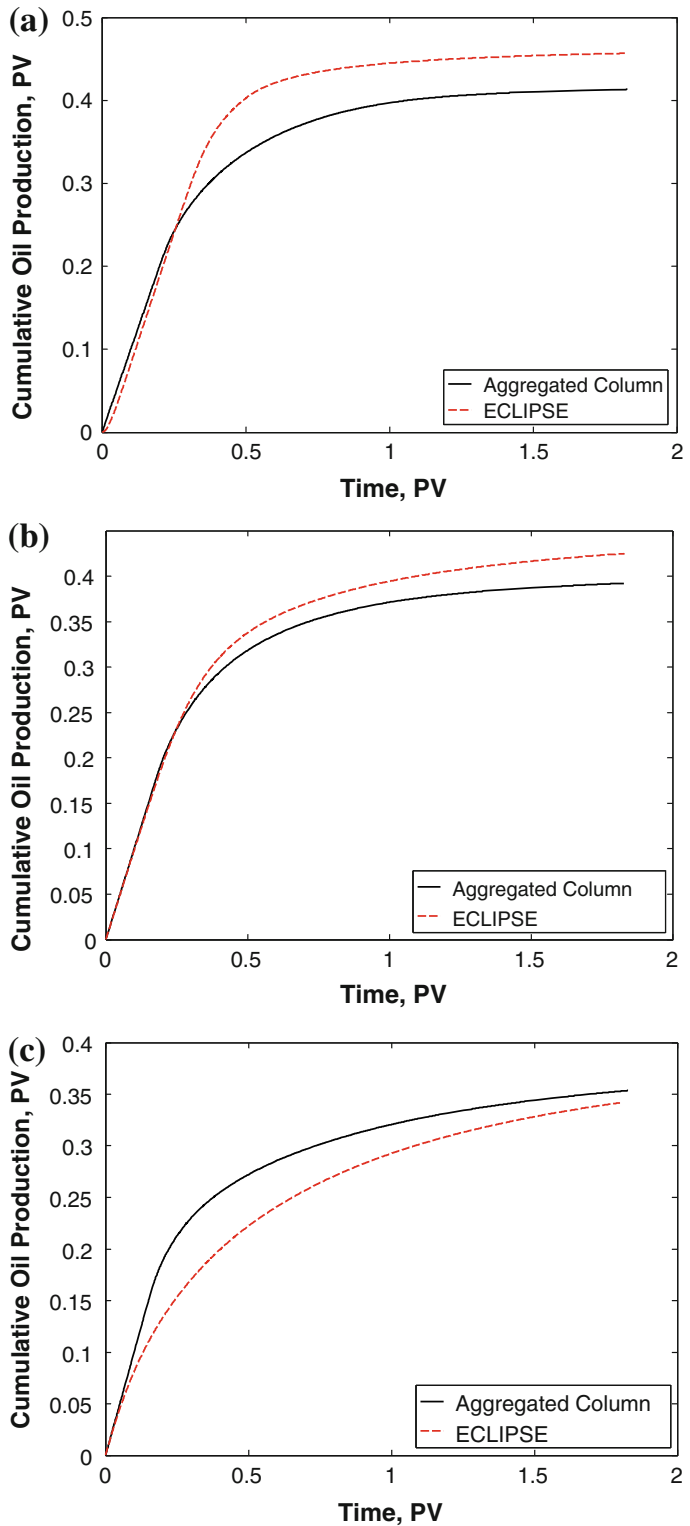

there is a critical Peclet number marking the boundary between the two regimes. If the Peclet number is large (as in Figs. 12b and 13c), there is a large difference between the homogenized model and either the effective permeability model or the BWR approach. In this regime, the oil recovery mechanism is controlled by the rate of imbibition from the matrix columns. In this case, the exchange rate between matrix and fracture is better described by the physically based homogenization approach than by the semi-empirical BWR approach. Therefore, we must use the aggregated column model when the Peclet number is large. 


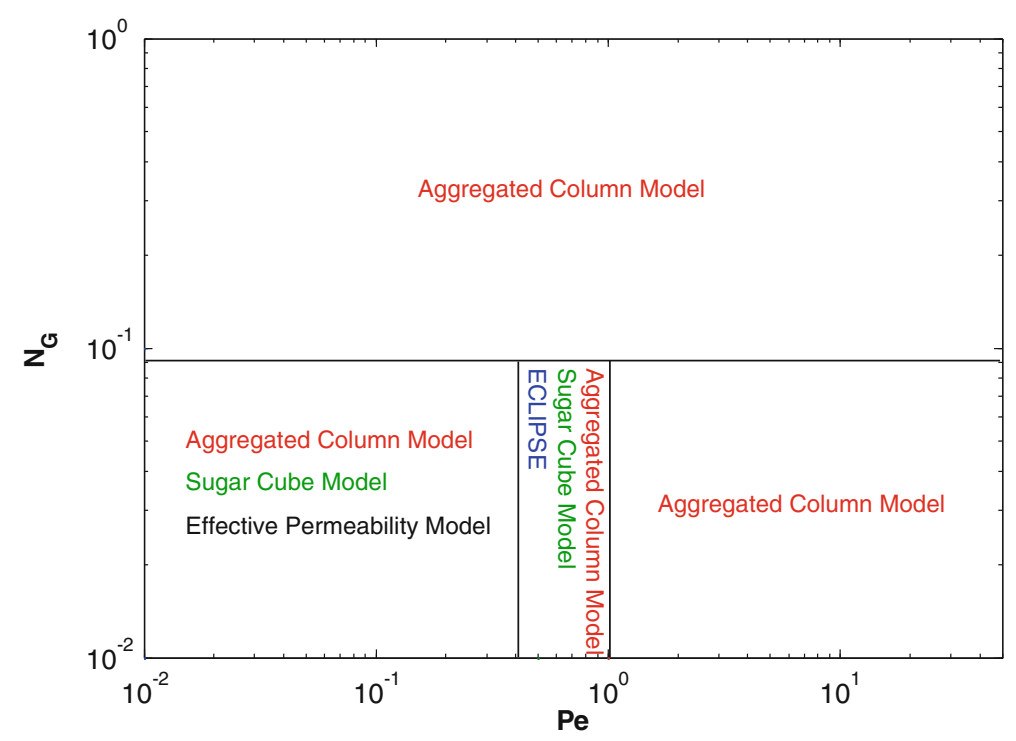

Fig. 14 A schematic parameter-space diagram showing which of the four models can be used for oil recovery prediction in vertically fractured reservoirs

Figure 14 summarizes the validity domain in the Peclet-gravity number space for the four models discussed in this article as to prediction of oil recovery factor in vertically fractured reservoirs.

\section{Conclusion}

- We obtain an upscaled equation based on the homogenization method for waterflooding vertically fractured oil reservoirs.

- We have developed an efficient numerical method to implement the upscaled equation in a computer code.

- When the Peclet number is small, the oil recovery mechanism is controlled by the rate of fluid transport in the fracture.

- When the Peclet number is large, the oil recovery mechanism is controlled by the rate of imbibition in the matrix columns.

- For a low injection rate, the matrix column size does not affect the oil production qualitatively. On the other hand, for a high injection rate, the matrix column size influences oil recovery factor significantly.

- For a small matrix column size, the injection rate does not affect the oil recovery factor qualitatively. However, for a big matrix column size, the injection rate influences oil recovery factor significantly.

- We distinguish two different practical production scenarios. (1) If a fractured reservoir has a large heterogeneity in terms of the matrix column size (fracture spacing), the best strategy to have a high oil recovery factor is to use a low water injection rate. (2) If a fractured reservoir contains matrix columns with a relatively small size, the best strategy 
to have a high net present value as well as a relatively high oil recovery factor is to use a high water injection rate.

- At low gravity numbers, gravity does not affect oil production. On the other hand, at intermediate and large gravity numbers considering gravity is necessary.

- The results show that in spite of the difference between the fluid-flow exchange term in the homogenized sugar cube model and the fluid-flow exchange term in the homogenized aggregated column model, the cumulative oil productions predicted by these two models are almost the same at low Peclet numbers. However, at large Peclet numbers, the reservoir topology needs to be considered precisely.

- When the Peclet number is low, the vertically fractured reservoir behaves like a highly heterogeneous single-porosity reservoir. Therefore, the effective permeability model can replace the aggregated column model without appreciable loss of accuracy. The advantage of the effective permeability model is again that its computations are not time-consuming as opposed to the aggregated column model.

- At a low Peclet number, gravity starts dominating; therefore, the aggregated column model cannot be replaced by the BWR approach because in the vertically fractured reservoir (aggregated column) gravity plays an important role. However, at intermediate Peclet numbers, one can use the BWR model to predict oil recovery factor from waterflooded vertically fractured reservoirs without loss of accuracy. On the other hand, at high Peclet numbers the aggregated column model, which represents the physics of the problem better than the BWR approach, should be used.

- In view of homogenization's physical basis, we assert that improved fracture modeling can be achieved using the upscaled aggregated column model. Figure 14 presents a "phase diagram" that can be used as an indication for the appropriate model or indicates when traditional modeling is adequate.

Acknowledgments We thank Statoil-Hydro for supporting our research on oil recovery from fractured reservoirs. We also thank Sharif University of Technology for their steady collaboration. We acknowledge Maryam Namdar Zanganeh, William R. Rossen, Stefan M. Luthi, and Giovanni Bertotti for many useful discussions and comments. We also acknowledge SPE for granting the Nico van Wingen Memorial Graduate Fellowship in Petroleum Engineering to Hamidreza Salimi.

Open Access This article is distributed under the terms of the Creative Commons Attribution Noncommercial License which permits any noncommercial use, distribution, and reproduction in any medium, provided the original author(s) and source are credited.

\section{References}

Aguilera, R.: Geological aspects of naturally fractured reservoirs. Lead. Age 17(12), 1667-1670 (1998)

AI-Harbi, M., Cheng, H., He, Z., Datta-Gupta, A.: Streamline-based production data integration in naturally fractured reservoirs. Soc. Petrol. Eng. J. 10(4), 426-439 (2005)

Al-Huthali, A., Datta-Gupta, A.: Streamline simulation of counter-current imbibition in naturally fractured reservoirs. J. Petrol. Sci. Eng. 43(3-4), 271-300 (2004)

Arbogast, T.: Gravitational forces in dual-porosity systems: I. Model derivation by homogenization. Transp. Porous Media 13(2), 179-203 (1993a)

Arbogast, T.: Gravitational Forces in Dual-Porosity Systems: II. Computational Validation of the Homogenized Model. Transp. Porous Media 13(2), 205-220 (1993b)

Arbogast, T.: Computational aspects of dual-porosity models. In: Hornung, U. (ed.) Homogenization and Porous Media, Interdisciplinary Applied Mathematics, 6th edn, pp. 203-223. Springer, New York (1997)

Arbogast, T., Douglas, J. Jr., Hornung, U.: Derivation of the double porosity model of single phase flow via homogenization theory. SIAM J. Math. Anal. 21(4), 823-836 (1990) 
Barenblatt, G.I., Zheltov, I.P., Kochina, I.N.: Basic concepts in the theory of seepage of homogenous liquids in fissured rocks. J. Appl. Math. Mech. 24(5), 852-864 (1960)

Berkowitz, B., Kosakowski, G., Margolin, G., Scher, H.: Application of continuous time random walk theory to tracer test measurements in fractured and heterogeneous porous media. Ground Water 39(4), 593604 (2001)

Chen, Z.: Homogenization and simulation for compositional flow in naturally fractured reservoirs. J. Math. Anal. Appl. 326(1), 12-32 (2007)

De Dreuzy, J.-R., Davy, P., Bour, O.: Hydraulic properties of two-dimensional random fracture networks following power law distributions of length and aperture. Water Resour. Res. 38(12), 121-129 (2002)

De Dreuzy, J.-R., Darcel, C., Davy, P., Bour, O.: Influence of spatial correlation of fracture centers on the permeability of two-dimensional fracture networks following a power law length distribution. Water Resour. Res. 40(1), W015021-W0150211 (2004)

Di Donato, G., Blunt, M.: Streamline-based dual-porosity simulation of reactive transport and flow in fractured reservoirs. Water Resour. Res. 40(4), W042031-W0420312 (2004)

Douglas, J. Jr., Hensley, J.L., Arbogast, T.: A dual-porosity model for waterflooding in naturally fractured reservoirs. Comput. Methods Appl. Mech. Eng. 87(2-3), 157-174 (1991)

Douglas, J. Jr., Arbogast, T., Paes-Leme, P.J., Hensley, J.L., Hunes, N.P.: Immiscible displacement in vertical fractured reservoirs. Transp. Porous Media 12(1), 73-106 (1993)

Firoozabadi, A.: Recovery mechanisms in fractured reservoirs and field performance. J. Can. Petrol. Technol. 39(11), 13-17 (2000)

Hornung, U.: Homogenization and Porous Media, Interdisciplinary Applied Mathematics, 6th edn. Springer, New York (1997)

Hoteit, H., Firoozabadi, A.: Compositional modeling of discrete-fractured media without transfer functions by the discontinuous galerkin and mixed methods. Soc. Petrol. Eng. J. 11(3), 341-352 (2006)

Kazemi, H., Seth, M.S., Thomas, G.W.: The interpretation of interference tests in naturally fractured reservoirs with uniform fracture distribution. Soc. Petrol. Eng. J. 9(4), 463-472 (1969)

Kazemi, H., Gilman, J.R., Elasharkawy, A.M.: Analytical and numerical solution of oil recovery from fractured reservoirs with empirical transfer functions. Soc. Petrol. Eng. Reserv. Eng. 7(2), 219-227 (1992)

Lewandowska, J., Szymkiewicz, A., Auriault, J.L.: Upscaling of Richards' equation for soils containing highly conductive inclusions. Adv. Water Resour. 28, 1159-1170 (2005)

Margolin, G., Berkowitz, B., Scher, H.: Structure, flow, and generalized conductivity scaling in fracture networks. Water Resour. Res. 34(9), 2103-2121 (1998)

Nelson, R.A.: Geologic Analysis of Naturally Fractured Reservoirs. Gulf Publishing Co., Houston, TX (1985)

Park, Y.-J., de Dreuzy, J.-R., Lee, K.-K., Berkowitz, B.: Transport and intersection mixing in random fracture networks with power law length distributions. Water Resour. Res. 37(10), 2493-2501 (2001)

Pooladi-Darvish, M., Firoozabadi, A.: Cocurrent and countercurrent imbibition in a water-wet matrix block. Soc. Petrol. Eng. J. 5(1), 3-11 (2000)

Salimi, H., Bruining, J.: Improved prediction of oil recovery from waterflooded fractured reservoirs using homogenization. Soc. Petrol. Eng. Reserv. Eng. Eval. (2009). doi:10.2118/115361-PA

Sarma, P., Aziz, K.: New transfer function for simulation of naturally fractured reservoirs with dual-porosity models. Soc. Petrol. Eng. J. 11(3), 328-340 (2006)

Shook, M., Li, D., Lake, L.W.: Scaling immiscible flow through permeable media by inspectional analysis. In Situ 16(4), 311-349 (1992)

Sonier, F., Souillard, P., Blaskovich, F.T. : Numerical simulation of naturally fractured reservoirs. Soc. Petrol. Eng. Reserv. Eng. 3(4), 1114-1122 (1988)

Tartar, L.: Incompressible fluid flow in a porous medium-convergence of the homogenization process. In: Sanchez-Palencia, E. (ed.) Lecture Notes in Physics, vol. 127, pp. 368-377. Springer, New York (1980)

Van Duijn, C.J., Molenaar, J., Neef, M.J.: The effect of capillary forces on immiscible two-phase flow in heterogeneous porous media. Transp. Porous Media 21(1), 71-93 (1995)

Van Duijn, C.J., Mikelić, A., Pop, I.S.: Effective equations for two-phase flow with trapping on the micro scale. SIAM J. Appl. Math. 62(5), 1531-1562 (2002)

Van Duijn, C.J., Mikelić, A., Pop, I.S., Rosier, C.: Effective dispersion equations for reactive flows with dominant Péclet and Damkohler numbers. Adv. Chem. Eng. 34, 1-45 (2008)

Warren, J.E., Root, P.J.: The behavior of naturally fractured reservoirs. Soc. Petrol. Eng. J. 3(3), 245-255 (1963)

Wu, Y.S., Pan, L., Pruess, K.: A physically based approach for modeling multiphase fracture-matrix interaction in fractured porous media. Adv. Water Resour. 27, 875-887 (2004)

Yortsos, Y.C.: A theoretical analysis of vertical flow equilibrium. Paper SPE 22612 presented at the SPE annual technical conference and exhibition, Dallas, TX, 6-9 October 1991. doi:10.2118/22612-MS 